\title{
Homogenization of Steklov Spectral Problems With INDEFINITE DENSITY FunCTION in Perforated Domains
}

\author{
Hermann Douanla* \\ Department of Mathematical Sciences \\ Chalmers University of Technology \\ Gothenburg, SE-41296, Sweden
}

\begin{abstract}
The asymptotic behavior of second order self-adjoint elliptic Steklov eigenvalue problems with periodic rapidly oscillating coefficients and with indefinite (sign-changing) density function is investigated in periodically perforated domains. We prove that the spectrum of this problem is discrete and consists of two sequences, one tending to $-\infty$ and another to $+\infty$. The limiting behavior of positive and negative eigencouples depends crucially on whether the average of the weight over the surface of the reference hole is positive, negative or equal to zero. By means of the two-scale convergence method, we investigate all three cases.
\end{abstract}

AMS Subject Classification:35B27, 35B40, 45C05.

Keywords: Homogenization, eigenvalue problems, perforated domains, indefinite weight function, two-scale convergence.

\section{Introduction}

In 1902, with a motivation coming from Physics, Steklov[33] introduced the following problem

$$
\left\{\begin{array}{l}
\Delta u=0 \quad \text { in } \Omega \\
\frac{\partial u}{\partial n}=\rho \lambda u \quad \text { on } \partial \Omega,
\end{array}\right.
$$

where $\lambda$ is a scalar and $\rho$ is a density function. The function $u$ represents the steady state temperature on $\Omega$ such that the flux on the boundary $\partial \Omega$ is proportional to the temperature. In two dimensions, assuming $\rho=1$, problem 1.1) can also be interpreted as a membrane with whole mass concentrated on the boundary. This problem has been later referred to as Steklov eigenvalue problem (Steklov is often transliterated as "Stekloff"). Moreover, eigenvalue problems also arise from many nonlinear problems after linearization (see e.g., the work of Hess and Kato[15, 16] and that of de Figueiredo[13]). This paper deals with the

${ }^{*}$ E-mail address: douanla@chalmers.se 
limiting behavior of a sequence of second order elliptic Steklov eigenvalue problems with indefinite(sign-changing) density function in perforated domains.

Let $\Omega$ be a bounded domain in $\mathbb{R}_{x}^{N}$ (the numerical space of variables $x=\left(x_{1}, \ldots, x_{N}\right)$ ), with $C^{1}$ boundary $\partial \Omega$ and with integer $N \geq 2$. We define the perforated domain $\Omega^{\varepsilon}$ as follows. Let $T \subset Y=(0,1)^{N}$ be a compact subset of $Y$ with $C^{1}$ boundary $\partial T(\equiv S)$ and nonempty interior. For $\varepsilon>0$, we define

$$
\begin{gathered}
t^{\varepsilon}=\left\{k \in \mathbb{Z}^{N}: \varepsilon(k+T) \subset \Omega\right\} \\
T^{\varepsilon}=\bigcup_{k \in t^{\varepsilon}} \varepsilon(k+T)
\end{gathered}
$$

and

$$
\Omega^{\varepsilon}=\Omega \backslash T^{\varepsilon} .
$$

In this setup, $T$ is the reference hole whereas $\varepsilon(k+T)$ is a hole of size $\varepsilon$ and $T^{\varepsilon}$ is the collection of the holes of the perforated domain $\Omega^{\varepsilon}$. The family $T^{\varepsilon}$ is made up with a finite number of holes since $\Omega$ is bounded. In the sequel, $Y^{*}$ stands for $Y \backslash T$ and $n=\left(n_{i}\right)_{i=1}^{N}$ denotes the outer unit normal vector to $S$ with respect to $Y^{*}$.

We are interested in the spectral asymptotics (as $\varepsilon \rightarrow 0$ ) of the following Steklov eigenvalue problem

$$
\left\{\begin{aligned}
-\sum_{i, j=1}^{N} \frac{\partial}{\partial x_{i}}\left(a_{i j}\left(\frac{x}{\varepsilon}\right) \frac{\partial u_{\varepsilon}}{\partial x_{j}}\right) & =0 \text { in } \Omega^{\varepsilon} \\
\sum_{i, j=1}^{N} a_{i j}\left(\frac{x}{\varepsilon}\right) \frac{\partial u_{\varepsilon}}{\partial x_{j}} n_{i}\left(\frac{x}{\varepsilon}\right) & =\rho\left(\frac{x}{\varepsilon}\right) \lambda_{\varepsilon} u_{\varepsilon} \text { on } \partial T^{\varepsilon} \\
u_{\varepsilon} & =0 \text { on } \partial \Omega
\end{aligned}\right.
$$

where $a_{i j} \in L^{\infty}\left(\mathbb{R}_{y}^{N}\right)(1 \leq i, j \leq N)$, with the symmetry condition $a_{j i}=a_{i j}$, the $Y$-periodicity hypothesis: for every $k \in \mathbb{Z}^{N}$ one has $a_{i j}(y+k)=a_{i j}(y)$ almost everywhere in $y \in \mathbb{R}_{y}^{N}$, and finally the (uniform) ellipticity condition: there exists $\alpha>0$ such that

$$
\sum_{i, j=1}^{N} a_{i j}(y) \xi_{j} \xi_{i} \geq \alpha|\xi|^{2}
$$

for all $\xi \in \mathbb{R}^{N}$ and for almost all $y \in \mathbb{R}_{y}^{N}$, where $|\xi|^{2}=\left|\xi_{1}\right|^{2}+\cdots+\left|\xi_{N}\right|^{2}$. The density function $\rho \in \mathcal{C}_{\text {per }}(Y)$ changes sign on $S$, that is, both the set $\{y \in S, \rho(y)<0\}$ and $\{y \in$ $S, \rho(y)>0\}$ are of positive $N-1$ dimensional Hausdorf measure (the so-called surface measure). This hypothesis makes the problem under consideration nonstandard. We will see (Corollary 2.15) that under the preceding hypotheses, for each $\varepsilon>0$ the spectrum of (1.2) is discrete and consists of two infinite sequences

$$
0<\lambda_{\varepsilon}^{1,+} \leq \lambda_{\varepsilon}^{2,+} \leq \cdots \leq \lambda_{\varepsilon}^{n,+} \leq \ldots, \quad \lim _{n \rightarrow+\infty} \lambda_{\varepsilon}^{n,+}=+\infty
$$

and

$$
0>\lambda_{\varepsilon}^{1,-} \geq \lambda_{\varepsilon}^{2,-} \geq \cdots \geq \lambda_{\varepsilon}^{n,-} \geq \ldots, \quad \lim _{n \rightarrow+\infty} \lambda_{\varepsilon}^{n,-}=-\infty .
$$


The asymptotic behavior of the eigencouples depends crucially on whether the average of the density $\rho$ over $S, M_{S}(\rho)=\int_{S} \rho(y) d \sigma(y)$, is positive, negative or equal to zero. All three cases are carefully investigated in this paper.

The homogenization of spectral problems has been widely explored. In a fixed domain, homogenization of spectral problems with point-wise positive density function goes back to Kesavan [18, 19]. Spectral asymptotics in perforated domains was studied by Vanninathan[35] and later in many other papers, including [11, 12, 17, 28, 29, 32] and the references therein. Homogenization of elliptic operators with sing-changing density function in a fixed domain with Dirichlet boundary conditions has been investigated by Nazarov et al. [22, 23, 24] via a combination of formal asymptotic expansion with Tartar's energy method. In porous media, spectral asymptotics of elliptic operator with sign changing density function is studied in [10] with the two scale convergence method.

The asymptotics of Steklov eigenvalue problems in periodically perforated domains was studied in [35] for the laplace operator and constant density $(\rho=1)$ using asymptotic expansion and Tartar's test function method. The same problem for a second order periodic elliptic operator has been studied in [29] (with $C^{\infty}$ coefficients) and in [11] (with $L^{\infty}$ coefficient) but still with constant density $(\rho=1)$. All the just-cited works deal only with one sequence of positive eigenvalues.

In this paper we take it to the general tricky step. We investigate in periodically perforated domains the asymptotic behavior of Steklov eigenvalue problems for periodic elliptic linear differential operators of order two in divergence form with $L^{\infty}$ coefficients and a sing-changing density function. We obtain accurate and concise homogenization results in all three cases: $M_{S}(\rho)>0$ (Theorem 3.1 and Theorem 3.3), $M_{S}(\rho)=0$ (Theorem 3.5,,$M_{S}(\rho)<0$ (Theorem 3.1 and Theorem 3.3), by using the two-scale convergence method[1, 21, 25, 36] introduced by Nguetseng[25] and further developed by Allaire[1]. In short;

i) If $M_{S}(\rho)>0$, then the positive eigencouples behave like in the case of point-wise positive density function, i.e., for $k \geq 1, \lambda_{\varepsilon}^{k,+}$ is of order $\varepsilon$ and $\frac{1}{\varepsilon} \lambda_{\varepsilon}^{k,+}$ converges as $\varepsilon \rightarrow 0$ to the $k^{\text {th }}$ eigenvalue of the limit Dirichlet spectral problem, corresponding extended eigenfunctions converge along subsequences.

As regards the "negative" eigencouples, $\lambda_{\varepsilon}^{k,-}$ converges to $-\infty$ at the rate $\frac{1}{\varepsilon}$ and the corresponding eigenfunctions oscillate rapidly. We use a factorization technique ([20, 35]) to prove that

$$
\lambda_{\varepsilon}^{k,-}=\frac{1}{\varepsilon} \lambda_{1}^{-}+\xi_{\varepsilon}^{k,-}+o(1), \quad k=1,2 \cdots
$$

where $\left(\lambda_{1}^{-}, \theta_{1}^{-}\right)$is the first negative eigencouple to the following local Steklov spectral problem

$$
\left\{\begin{array}{c}
-\operatorname{div}\left(a(y) D_{y} \theta\right)=0 \quad \text { in } Y^{*} \\
a(y) D_{y} \theta \cdot n=\lambda \rho(y) \theta \quad \text { on } S \\
\theta \quad Y-\text { periodic, }
\end{array}\right.
$$

and $\left\{\xi_{\varepsilon}^{k, \pm}\right\}_{k=1}^{\infty}$ are eigenvalues of a Steklov eigenvalue problem similar to (1.2). We then prove that $\left\{\frac{\lambda_{\varepsilon}^{k,-}}{\varepsilon}-\frac{\lambda_{1}^{-}}{\varepsilon^{2}}\right\}$ converges to the $k^{\text {th }}$ eigenvalue of a limit Dirichlet spectral 
problem which is different from that obtained for positive eigenvalues. As regards eigenfunctions, extensions of $\left\{\frac{u_{\varepsilon}^{k,-}}{\left(\theta_{1}^{-}\right)^{\varepsilon}}\right\}_{\varepsilon \in E}$ - where $\left(\theta_{1}^{-}\right)^{\varepsilon}(x)=\theta_{1}^{-}\left(\frac{x}{\varepsilon}\right)$ - converge along subsequences to the $k^{\text {th }}$ eigenfunctions of the limit problem.

ii) If $M_{S}(\rho)=0$, then the limit spectral problem generates a quadratic operator pencil and $\lambda_{\varepsilon}^{k, \pm}$ converges to the $(k, \pm)^{t h}$ eigenvalue of the limit operator, extended eigenfunctions converge along subsequences as well. This case requires a new convergence result as regards the two-scale convergence theory, Lemma 2.9

iii) The case when $M_{S}(\rho)<0$ is equivalent to that when $M_{S}(\rho)>0$, just replace $\rho$ with $-\rho$.

Unless otherwise specified, vector spaces throughout are considered over $\mathbb{R}$, and scalar functions are assumed to take real values. We will make use of the following notations. Let $F\left(\mathbb{R}^{N}\right)$ be a given function space. We denote by $F_{\text {per }}(Y)$ the space of functions in $F_{\text {loc }}\left(\mathbb{R}^{N}\right)$ (when it makes sense) that are $Y$-periodic, and by $F_{\text {per }}(Y) / \mathbb{R}$ the space of those functions $u \in F_{p e r}(Y)$ with $\int_{Y} u(y) d y=0$. We denote by $H_{p e r}^{1}\left(Y^{*}\right)$ the space of functions in $H^{1}\left(Y^{*}\right)$ assuming same values on the opposite faces of $Y$ and $H_{p e r}^{1}\left(Y^{*}\right) / \mathbb{R}$ stands for the subset of $H_{p e r}^{1}\left(Y^{*}\right)$ made up of functions $u \in H_{p e r}^{1}\left(Y^{*}\right)$ verifying $\int_{Y^{*}} u(y) d y=0$. Finally, the letter $E$ denotes throughout a family of strictly positive real numbers $(0<\varepsilon<1)$ admitting 0 as accumulation point. The numerical space $\mathbb{R}^{N}$ and its open sets are provided with the Lebesgue measure denoted by $d x=d x_{1} \ldots d x_{N}$. The usual gradient operator will be denoted by $D$. For the sake of simple notations we hide trace operators. The rest of the paper is organized as follows. Section 2 deals with some preliminary results while homogenization processes are considered in Section 3 .

\section{Preliminaries}

We first recall the definition and the main compactness theorems of the two-scale convergence method. Let $\Omega$ be a smooth open bounded set in $\mathbb{R}_{x}^{N}$ (integer $N \geq 2$ ) and $Y=(0,1)^{N}$, the unit cube.

Definition 2.1. A sequence $\left(u_{\varepsilon}\right)_{\varepsilon \in E} \subset L^{2}(\Omega)$ is said to two-scale converge in $L^{2}(\Omega)$ to some $u_{0} \in L^{2}(\Omega \times Y)$ if as $E \ni \varepsilon \rightarrow 0$,

$$
\int_{\Omega} u_{\varepsilon}(x) \phi\left(x, \frac{x}{\varepsilon}\right) d x \rightarrow \iint_{\Omega \times Y} u_{0}(x, y) \phi(x, y) d x d y
$$

for all $\phi \in L^{2}\left(\Omega ; C_{p e r}(Y)\right)$.

Notation. We express this by writing $u_{\varepsilon} \stackrel{2 s}{\rightarrow} u_{0}$ in $L^{2}(\Omega)$.

The following compactness theorems [1, 25, 27] are cornerstones of the two-scale convergence method.

Theorem 2.2. Let $\left(u_{\varepsilon}\right)_{\varepsilon \in E}$ be a bounded sequence in $L^{2}(\Omega)$. Then a subsequence $E^{\prime}$ can be extracted from $E$ such that as $E^{\prime} \ni \varepsilon \rightarrow 0$, the sequence $\left(u_{\varepsilon}\right)_{\varepsilon \in E^{\prime}}$ two-scale converges in $L^{2}(\Omega)$ to some $u_{0} \in L^{2}(\Omega \times Y)$. 
Theorem 2.3. Let $\left(u_{\varepsilon}\right)_{\varepsilon \in E}$ be a bounded sequence in $H^{1}(\Omega)$. Then a subsequence $E^{\prime}$ can be extracted from $E$ such that as $E^{\prime} \ni \varepsilon \rightarrow 0$

$$
\begin{aligned}
u_{\varepsilon} & \rightarrow u_{0} \quad \text { in } H^{1}(\Omega) \text {-weak } \\
u_{\varepsilon} & \rightarrow u_{0} \quad \text { in } L^{2}(\Omega) \\
\frac{\partial u_{\varepsilon}}{\partial x_{j}} & \stackrel{2 s}{\rightarrow} \frac{\partial u_{0}}{\partial x_{j}}+\frac{\partial u_{1}}{\partial y_{j}} \quad \text { in } L^{2}(\Omega) \quad(1 \leq j \leq N)
\end{aligned}
$$

where $u_{0} \in H^{1}(\Omega)$ and $u_{1} \in L^{2}\left(\Omega ; H_{p e r}^{1}(Y)\right)$. Moreover, as $E^{\prime} \ni \varepsilon \rightarrow 0$ we have

$$
\int_{\Omega} \frac{u_{\varepsilon}(x)}{\varepsilon} \psi\left(x, \frac{x}{\varepsilon}\right) d x \rightarrow \iint_{\Omega \times Y} u_{1}(x, y) \psi(x, y) d x d y
$$

for $\psi \in \mathcal{D}(\Omega) \otimes\left(L_{\text {per }}^{2}(Y) / \mathbb{R}\right)$.

Remark 2.4. In Theorem 2.3 the function $u_{1}$ is unique up to an additive function of variable $x$. We need to fix its choice according to our future needs. To do this, we introduce the following space

$$
H_{p e r}^{1, *}(Y)=\left\{u \in H_{p e r}^{1}(Y): \int_{Y^{*}} u(y) d y=0\right\} .
$$

This defines a closed subspace of $H_{p e r}^{1}(Y)$ as it is the kernel of the bounded linear functional $u \mapsto \int_{Y^{*}} u(y) d y$ defined on $H_{p e r}^{1}(Y)$. It is to be noted that for $u \in H_{p e r}^{1, *}(Y)$, its restriction to $Y^{*}$ (which will still be denoted by $u$ in the sequel) belongs to $H_{p e r}^{1}\left(Y^{*}\right) / \mathbb{R}$.

We will use the following version of Theorem 2.3 .

Theorem 2.5. Let $\left(u_{\varepsilon}\right)_{\varepsilon \in E}$ be a bounded sequence in $H^{1}(\Omega)$. Then a subsequence $E^{\prime}$ can be extracted from $E$ such that as $E^{\prime} \ni \varepsilon \rightarrow 0$

$$
\begin{aligned}
u_{\varepsilon} & \rightarrow u_{0} \quad \text { in } H^{1}(\Omega) \text {-weak } \\
u_{\varepsilon} & \rightarrow u_{0} \quad \text { in } L^{2}(\Omega) \\
\frac{\partial u_{\varepsilon}}{\partial x_{j}} & \stackrel{2 s}{\rightarrow} \frac{\partial u_{0}}{\partial x_{j}}+\frac{\partial u_{1}}{\partial y_{j}} \quad \text { in } L^{2}(\Omega) \quad(1 \leq j \leq N)
\end{aligned}
$$

where $u_{0} \in H^{1}(\Omega)$ and $u_{1} \in L^{2}\left(\Omega ; H_{\text {per }}^{1, *}(Y)\right)$. Moreover, as $E^{\prime} \ni \varepsilon \rightarrow 0$ we have

$$
\int_{\Omega} \frac{u_{\varepsilon}(x)}{\varepsilon} \psi\left(x, \frac{x}{\varepsilon}\right) d x \rightarrow \iint_{\Omega \times Y} u_{1}(x, y) \psi(x, y) d x d y
$$

for $\psi \in \mathcal{D}(\Omega) \otimes\left(L_{\text {per }}^{2}(Y) / \mathbb{R}\right)$.

Proof. Let $\widetilde{u}_{1} \in L^{2}\left(\Omega ; H_{p e r}^{1}(Y)\right)$ be such that Theorem 2.3 holds with $\widetilde{u}_{1}$ in place of $u_{1}$. Put

$$
u_{1}(x, y)=\widetilde{u}_{1}(x, y)-\frac{1}{\left|Y^{*}\right|} \int_{Y^{*}} \widetilde{u}_{1}(x, y) d y \quad(x, y) \in \Omega \times Y
$$

where $\left|Y^{*}\right|$ stands for the Lebesgue measure of $Y^{*}$. Then $u_{1} \in L^{2}\left(\Omega ; H_{\text {per }}^{1, *}(Y)\right)$ and moreover $D_{y} u_{1}=D_{y} \widetilde{u}_{1}$ so that 2.5 holds. 
In the sequel, $S^{\varepsilon}$ stands for $\partial T^{\varepsilon}$ and the surface measures on $S$ and $S^{\varepsilon}$ are denoted by $d \sigma(y)(y \in Y), d \sigma_{\varepsilon}(x)(x \in \Omega, \varepsilon \in E)$, respectively. The space of squared integrable functions, with respect to the previous measures on $S$ and $S^{\varepsilon}$ are denoted by $L^{2}(S)$ and $L^{2}\left(S^{\varepsilon}\right)$ respectively. Since the volume of $S^{\varepsilon}$ grows proportionally to $\frac{1}{\varepsilon}$ as $\varepsilon \rightarrow 0$, we endow $L^{2}\left(S^{\varepsilon}\right)$ with the scaled scalar product [3, 30, 31]

$$
(u, v)_{L^{2}\left(S^{\varepsilon}\right)}=\varepsilon \int_{S^{\varepsilon}} u(x) v(x) d \sigma_{\varepsilon}(x) \quad\left(u, v \in L^{2}\left(S^{\varepsilon}\right)\right) .
$$

Definition 2.1 and theorem 2.2 then generalize as

Definition 2.6. A sequence $\left(u_{\varepsilon}\right)_{\varepsilon \in E} \subset L^{2}\left(S^{\varepsilon}\right)$ is said to two-scale converge to some $u_{0} \in$ $L^{2}(\Omega \times S)$ if as $E \ni \varepsilon \rightarrow 0$,

$$
\varepsilon \int_{S^{\varepsilon}} u_{\varepsilon}(x) \phi\left(x, \frac{x}{\varepsilon}\right) d \sigma_{\varepsilon}(x) \rightarrow \iint_{\Omega \times S} u_{0}(x, y) \phi(x, y) d x d \sigma(y)
$$

for all $\phi \in \mathcal{C}\left(\bar{\Omega} ; \mathcal{C}_{\text {per }}(Y)\right)$.

Theorem 2.7. Let $\left(u_{\varepsilon}\right)_{\varepsilon \in E}$ be a sequence in $L^{2}\left(S^{\varepsilon}\right)$ such that

$$
\varepsilon \int_{S^{\varepsilon}}\left|u_{\varepsilon}(x)\right|^{2} d \sigma_{\varepsilon}(x) \leq C
$$

where $C$ is a positive constant independent of $\varepsilon$. There exists a subsequence $E^{\prime}$ of $E$ such that $\left(u_{\varepsilon}\right)_{\varepsilon \in E^{\prime}}$ two-scale converges to some $u_{0} \in L^{2}\left(\Omega ; L^{2}(S)\right)$ in the sense of definition 2.6

In the case when $\left(u_{\varepsilon}\right)_{\varepsilon \in E}$ is the sequence of traces on $S^{\varepsilon}$ of functions in $H^{1}(\Omega)$, one can link its usual two-scale limit with its surface two-scale limits. The following proposition whose proof can be found in [3] clarifies this.

Proposition 2.8. Let $\left(u_{\varepsilon}\right)_{\varepsilon \in E} \subset H^{1}(\Omega)$ be such that

$$
\left\|u_{\varepsilon}\right\|_{L^{2}(\Omega)}+\varepsilon\left\|D u_{\varepsilon}\right\|_{L^{2}(\Omega)^{N}} \leq C,
$$

where $C$ is a positive constant independent of $\varepsilon$ and $D$ denotes the usual gradient. The sequence of traces of $\left(u_{\varepsilon}\right)_{\varepsilon \in E}$ on $S^{\varepsilon}$ satisfies

$$
\varepsilon \int_{S^{\varepsilon}}\left|u_{\varepsilon}(x)\right|^{2} d \sigma_{\varepsilon}(x) \leq C \quad(\varepsilon \in E)
$$

and up to a subsequence $E^{\prime}$ of $E$, it two-scale converges in the sense of Definition 2.6 to some $u_{0} \in L^{2}\left(\Omega ; L^{2}(S)\right)$ which is nothing but the trace on $S$ of the usual two-scale limit, a function in $L^{2}\left(\Omega ; H_{\text {per }}^{1}(Y)\right)$. More precisely, as $E^{\prime} \ni \varepsilon \rightarrow 0$

$$
\begin{aligned}
\varepsilon \int_{S^{\varepsilon}} u_{\varepsilon}(x) \phi\left(x, \frac{x}{\varepsilon}\right) d \sigma_{\varepsilon}(x) & \rightarrow \iint_{\Omega \times S} u_{0}(x, y) \phi(x, y) d x d \sigma(y), \\
\int_{\Omega} u_{\varepsilon}(x) \phi\left(x, \frac{x}{\varepsilon}\right) d x d y & \rightarrow \iint_{\Omega \times Y} u_{0}(x, y) \phi(x, y) d x d y,
\end{aligned}
$$

for all $\phi \in \mathcal{C}\left(\bar{\Omega} ; \mathcal{C}_{\text {per }}(Y)\right)$. 
In our homogenization process, more precisely in the case when $M_{S}(\rho)=0$, we will need a generalization of (2.2) to periodic surfaces. Notice that (2.2) was proved for the first time in a deterministic setting by Nguetseng and Woukeng in [27] but to the best of our knowledge its generalization to periodic surfaces is not yet available in the literature. We state and prove it below.

Lemma 2.9. Let $\left(u_{\varepsilon}\right)_{\varepsilon \in E} \subset H^{1}(\Omega)$ be such that as $E \ni \varepsilon \rightarrow 0$

$$
\begin{aligned}
u_{\varepsilon} & \stackrel{2 s}{\rightarrow} u_{0} \quad \text { in } L^{2}(\Omega) \\
\frac{\partial u_{\varepsilon}}{\partial x_{j}} & \stackrel{2 s}{\rightarrow} \frac{\partial u_{0}}{\partial x_{j}}+\frac{\partial u_{1}}{\partial y_{j}} \quad \text { in } L^{2}(\Omega) \quad(1 \leq j \leq N)
\end{aligned}
$$

for some $u_{0} \in H^{1}(\Omega)$ and $u_{1} \in L^{2}\left(\Omega ; H_{p e r}^{1}(Y)\right)$. Then

$$
\lim _{\varepsilon \rightarrow 0} \int_{S^{\varepsilon}} u_{\varepsilon}(x) \varphi(x) \theta\left(\frac{x}{\varepsilon}\right) d \sigma_{\varepsilon}(x)=\iint_{\Omega \times S} u_{1}(x, y) \varphi(x) \theta(y) d x d \sigma(y)
$$

for all $\varphi \in \mathcal{D}(\Omega)$ and $\theta \in \mathcal{C}_{\text {per }}(Y)$ with $\int_{S} \theta(y) d \sigma(y)=0$.

Proof. We first transform the above surface integral into a volume integral by adapting the trick in [7, Section 3]. By the mean value zero condition over $S$ for $\theta$ we conclude that there exists a unique solution $\vartheta \in H_{p e r}^{1}\left(Y^{*}\right) / \mathbb{R}$ to

$$
\left\{\begin{array}{l}
-\Delta_{y} \vartheta=0 \text { in } Y^{*} \\
D_{y} \vartheta(y) \cdot n(y)=\theta(y) \text { on } S,
\end{array}\right.
$$

where $n=\left(n_{i}\right)_{i=1}^{N}$ stands for the outward unit normal to $S$ with respect to $Y^{*}$. Put $\phi=D_{y} \vartheta$. We get

$$
\begin{aligned}
\int_{\Omega^{\varepsilon}} D_{x} u_{\varepsilon}(x) \varphi(x) & \cdot D_{y} \vartheta\left(\frac{x}{\varepsilon}\right) d x=\int_{S^{\varepsilon}} u_{\varepsilon}(x) \varphi(x) D_{y} \vartheta\left(\frac{x}{\varepsilon}\right) \cdot n\left(\frac{x}{\varepsilon}\right) d \sigma_{\varepsilon}(x) \\
& -\int_{\Omega^{\varepsilon}} u_{\varepsilon}(x) D_{x} \varphi(x) \cdot D_{y} \vartheta\left(\frac{x}{\varepsilon}\right) d x-\frac{1}{\varepsilon} \int_{\Omega^{\varepsilon}} u_{\varepsilon}(x) \varphi(x) \Delta_{y} \vartheta\left(\frac{x}{\varepsilon}\right) d x \\
= & \int_{S^{\varepsilon}} u_{\varepsilon}(x) \varphi(x) \theta\left(\frac{x}{\varepsilon}\right) d \sigma_{\varepsilon}(x)-\int_{\Omega^{\varepsilon}} u_{\varepsilon}(x) D_{x} \varphi(x) \cdot \phi\left(\frac{x}{\varepsilon}\right) d x
\end{aligned}
$$

Next, sending $\varepsilon$ to 0 yields

$$
\begin{aligned}
\lim _{\varepsilon \rightarrow 0} \int_{S^{\varepsilon}} u_{\varepsilon}(x) \varphi(x) \theta\left(\frac{x}{\varepsilon}\right) d \sigma_{\varepsilon}(x)= & \iint_{\Omega \times Y^{*}}\left[D_{x} u_{0}(x)+D_{y} u_{1}(x, y)\right] \varphi(x) \cdot \phi(y) d x d y \\
& +\iint_{\Omega \times Y^{*}} u_{0}(x) D_{x} \varphi(x) \cdot \phi(y) d x d y \\
= & \iint_{\Omega \times Y^{*}} D_{y} u_{1}(x, y) \varphi(x) \cdot \phi(y) d x d y .
\end{aligned}
$$

We finally have

$$
\begin{aligned}
\iint_{\Omega \times Y^{*}} D_{y} u_{1}(x, y) \varphi(x) \cdot \phi(y) d x d y & =-\iint_{\Omega \times Y^{*}} u_{1}(x, y) \varphi(x) \Delta_{y} \vartheta(y) d x d y \\
& +\iint_{\Omega \times S} u_{1}(x, y) \varphi(x) \phi(y) \cdot n(y) d x d \sigma(y) \\
& =\iint_{\Omega \times S} u_{1}(x, y) \varphi(x) \theta(y) d x d \sigma(y) .
\end{aligned}
$$


The proof is completed.

We now gather some preliminary results. We introduce the characteristic function $\chi_{G}$ of

$$
G=\mathbb{R}_{y}^{N} \backslash \Theta
$$

with

$$
\Theta=\bigcup_{k \in \mathbb{Z}^{N}}(k+T) .
$$

It is clear that $G$ is an open subset of $\mathbb{R}_{y}^{N}$. Next, let $\varepsilon \in E$ be arbitrarily fixed and define

$$
V_{\varepsilon}=\left\{u \in H^{1}\left(\Omega^{\varepsilon}\right): u=0 \text { on } \partial \Omega\right\} .
$$

We equip $V_{\varepsilon}$ with the $H^{1}\left(\Omega^{\varepsilon}\right)$-norm which makes it a Hilbert space. We recall the following classical extension result [8].

Proposition 2.10. For each $\varepsilon \in E$ there exists an operator $P_{\varepsilon}$ of $V_{\varepsilon}$ into $H_{0}^{1}(\Omega)$ with the following properties:

- $P_{\varepsilon}$ sends continuously and linearly $V_{\varepsilon}$ into $H_{0}^{1}(\Omega)$.

- $\left.\left(P_{\varepsilon} v\right)\right|_{\Omega^{\varepsilon}}=v$ for all $v \in V_{\varepsilon}$.

- $\left\|D\left(P_{\varepsilon} v\right)\right\|_{L^{2}(\Omega)^{N}} \leq c\|D v\|_{L^{2}\left(\Omega^{\varepsilon}\right)^{N}}$ for all $v \in V_{\varepsilon}$, where $c$ is a constant independent of $\varepsilon$.

In the sequel, we will explicitly write the just-defined extension operator everywhere needed but we will abuse notations on the local extension operator (see [8] for its definition): the extension to $Y$ of $u \in H_{\text {per }}^{1}\left(Y^{*}\right) / \mathbb{R}$ will still be denoted by $u$ (this extension is an element of $\left.H_{\text {per }}^{1, *}(Y)\right)$.

Now, let $Q^{\varepsilon}=\Omega \backslash(\varepsilon \Theta)$. This defines an open set in $\mathbb{R}^{N}$ and $\Omega^{\varepsilon} \backslash Q^{\varepsilon}$ is the intersection of $\Omega$ with the collection of the holes crossing the boundary $\partial \Omega$. The following result implies that the holes crossing the boundary $\partial \Omega$ are of no effects as regards the homogenization process.

Lemma 2.11. [26] Let $K \subset \Omega$ be a compact set independent of $\varepsilon$. There is some $\varepsilon_{0}>0$ such that $\Omega^{\varepsilon} \backslash Q^{\varepsilon} \subset \Omega \backslash K$ for any $0<\varepsilon \leq \varepsilon_{0}$.

We introduce the space

$$
\mathbb{F}_{0}^{1}=H_{0}^{1}(\Omega) \times L^{2}\left(\Omega ; H_{\text {per }}^{1, *}(Y)\right)
$$

and endow it with the following norm

$$
\|\mathbf{v}\|_{\mathbb{F}_{0}^{1}}=\left\|D_{x} v_{0}+D_{y} v_{1}\right\|_{L^{2}(\Omega \times Y)} \quad\left(\mathbf{v}=\left(v_{0}, v_{1}\right) \in \mathbb{F}_{0}^{1}\right),
$$

which makes it a Hilbert space admitting $F_{0}^{\infty}=\mathcal{D}(\Omega) \times\left[\mathcal{D}(\Omega) \otimes \mathcal{C}_{\text {per }}^{\infty, *}(Y)\right]\left(\right.$ where $\mathcal{C}_{\text {per }}^{\infty, *}(Y)=$ $\left.\left\{u \in \mathcal{C}_{\text {per }}^{\infty}(Y): \int_{Y^{*}} u(y) d y=0\right\}\right)$ as a dense subspace. For $(\mathbf{u}, \mathbf{v}) \in \mathbb{F}_{0}^{1} \times \mathbb{F}_{0}^{1}$, let

$$
a_{\Omega}(\mathbf{u}, \mathbf{v})=\sum_{i, j=1}^{N} \iint_{\Omega \times Y^{*}} a_{i j}(y)\left(\frac{\partial u_{0}}{\partial x_{j}}+\frac{\partial u_{1}}{\partial y_{j}}\right)\left(\frac{\partial v_{0}}{\partial x_{i}}+\frac{\partial v_{1}}{\partial y_{i}}\right) d x d y .
$$

This define a symmetric, continuous bilinear form on $\mathbb{F}_{0}^{1} \times \mathbb{F}_{0}^{1}$. We will need the following results whose proof can be found in [11]. 
Lemma 2.12. Fix $\Phi=\left(\psi_{0}, \psi_{1}\right) \in F_{0}^{\infty}$ and define $\Phi_{\varepsilon}: \Omega \rightarrow \mathbb{R}(\varepsilon>0)$ by

$$
\Phi_{\varepsilon}(x)=\psi_{0}(x)+\varepsilon \psi_{1}\left(x, \frac{x}{\varepsilon}\right) \quad(x \in \Omega) .
$$

If $\left(u_{\varepsilon}\right)_{\varepsilon \in E} \subset H_{0}^{1}(\Omega)$ is such that

$$
\frac{\partial u_{\varepsilon}}{\partial x_{i}} \stackrel{2 s}{\rightarrow} \frac{\partial u_{0}}{\partial x_{i}}+\frac{\partial u_{1}}{\partial y_{i}} \quad \text { in } \quad L^{2}(\Omega)(1 \leq i \leq N)
$$

as $E \ni \varepsilon \rightarrow 0$ for some $\boldsymbol{u}=\left(u_{0}, u_{1}\right) \in \mathbb{F}_{0}^{1}$, then

$$
a^{\varepsilon}\left(u_{\varepsilon}, \Phi_{\varepsilon}\right) \rightarrow a_{\Omega}(\boldsymbol{u}, \Phi)
$$

as $E \ni \varepsilon \rightarrow 0$, where

$$
a^{\varepsilon}\left(u_{\varepsilon}, \Phi_{\varepsilon}\right)=\sum_{i, j=1}^{N} \int_{\Omega^{\varepsilon}} a_{i j}\left(\frac{x}{\varepsilon}\right) \frac{\partial u_{\varepsilon}}{\partial x_{j}} \frac{\partial \Phi_{\varepsilon}}{\partial x_{i}} d x .
$$

We now construct and point out the main properties of the so-called homogenized coefficients. Put

$$
\begin{aligned}
& a(u, v)=\sum_{i, j=1}^{N} \int_{Y^{*}} a_{i j}(y) \frac{\partial u}{\partial y_{j}} \frac{\partial v}{\partial y_{i}} d y, \\
& l_{j}(v)=\sum_{k=1}^{N} \int_{Y^{*}} a_{k j}(y) \frac{\partial v}{\partial y_{k}} d y, \quad(1 \leq j \leq N)
\end{aligned}
$$

and

$$
l_{0}(v)=\int_{S} \rho(y) v(y) d \sigma(y),
$$

for $u, v \in H_{p e r}^{1}\left(Y^{*}\right) / \mathbb{R}$. Equipped with the norm

$$
\|u\|_{H_{p e r}^{1}\left(Y^{*}\right) / \mathbb{R}}=\left\|D_{y} u\right\|_{L^{2}\left(Y^{*}\right)^{N}} \quad\left(u \in H_{p e r}^{1}\left(Y^{*}\right) / \mathbb{R}\right),
$$

$H_{p e r}^{1}\left(Y^{*}\right) / \mathbb{R}$ is a Hilbert space.

Proposition 2.13. Let $1 \leq j \leq N$. The local variational problems

$$
u \in H_{p e r}^{1}\left(Y^{*}\right) / \mathbb{R} \text { and } a(u, v)=l_{j}(v) \text { for all } v \in H_{p e r}^{1}\left(Y^{*}\right) / \mathbb{R}
$$

and

$$
u \in H_{p e r}^{1}\left(Y^{*}\right) / \mathbb{R} \text { and } a(u, v)=l_{0}(v) \text { for all } v \in H_{p e r}^{1}\left(Y^{*}\right) / \mathbb{R}
$$

admit each a unique solution, assuming for (2.14) that $M_{S}(\rho)=0$.

Let $1 \leq i, j \leq N$. The homogenized coefficients read

$$
q_{i j}=\int_{Y^{*}} a_{i j}(y) d y-\sum_{l=1}^{N} \int_{Y^{*}} a_{i l}(y) \frac{\partial \chi^{j}}{\partial y_{l}}(y) d y
$$


where $\chi^{j} \quad(1 \leq j \leq N)$ is the solution to (2.13). We recall that $q_{j i}=q_{i j}(1 \leq i, j \leq N)$ and there exists a constant $\alpha_{0}>0$ such that

$$
\sum_{i, j=1}^{N} q_{i j} \xi_{j} \xi_{i} \geq \alpha_{0}|\xi|^{2}
$$

for all $\xi \in \mathbb{R}^{N}$ (see e.g., [4]).

We now visit the existence result for (1.2). The weak formulation of (1.2) reads: Find $\left(\lambda_{\varepsilon}, u_{\varepsilon}\right) \in \mathbb{C} \times V_{\varepsilon},\left(u_{\varepsilon} \neq 0\right)$ such that

$$
a^{\varepsilon}\left(u_{\varepsilon}, v\right)=\lambda_{\varepsilon}\left(\rho^{\varepsilon} u_{\varepsilon}, v\right)_{S^{\varepsilon}}, \quad v \in V_{\varepsilon},
$$

where

$$
\left(\rho^{\varepsilon} u_{\varepsilon}, v\right)_{S^{\varepsilon}}=\int_{S^{\varepsilon}} \rho^{\varepsilon} u_{\varepsilon} v d \sigma_{\varepsilon}(x) .
$$

Since $\rho^{\varepsilon}$ changes sign, the classical results on the spectrum of semi-bounded self-adjoint operators with compact resolvent do not apply. To handle this, we follow the ideas in [24]. The bilinear form $\left(\rho^{\varepsilon} u, v\right)_{S^{\varepsilon}}$ defines a bounded linear operator $K^{\varepsilon}: V_{\varepsilon} \rightarrow V_{\varepsilon}$ such that

$$
\left(\rho^{\varepsilon} u, v\right)_{S^{\varepsilon}}=a^{\varepsilon}\left(K^{\varepsilon} u, v\right) \quad\left(u, v \in V_{\varepsilon}\right) .
$$

The operator $K^{\varepsilon}$ is symmetric and its domains $D\left(K^{\varepsilon}\right)$ coincides with the whole $V_{\varepsilon}$, thus it is self-adjoint. Recall that the gradient norm is equivalent to the $H^{1}\left(\Omega^{\varepsilon}\right)$-norm on $V_{\varepsilon}$. Looking at $K^{\varepsilon} u$ as the solution to the boundary value problem

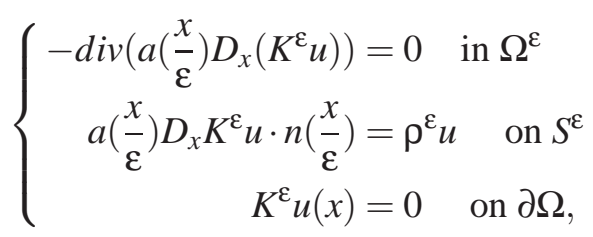

we get a constant $C_{\varepsilon}>0$ such that $\left\|K^{\varepsilon} u\right\|_{V^{\varepsilon}} \leq C_{\varepsilon}\|u\|_{L^{2}\left(S^{\varepsilon}\right)}$. But the trace operator $V_{\varepsilon} \rightarrow$ $L^{2}\left(S^{\varepsilon}\right)$ is compact. The compactness of $K^{\varepsilon}$ follows thereby. We can rewrite (2.16) as follows

$$
K^{\varepsilon} u_{\varepsilon}=\mu_{\varepsilon} u_{\varepsilon}, \quad \mu_{\varepsilon}=\frac{1}{\lambda_{\varepsilon}} .
$$

We recall that (see e.g., [5]) in the case $\rho \geq 0$ on $S$, the operator $K^{\varepsilon}$ is positive and its spectrum $\sigma\left(K^{\varepsilon}\right)$ lies in $\left[0,\left\|K^{\varepsilon}\right\|\right]$ and $\mu_{\varepsilon}=0$ belongs to the essential spectrum $\sigma_{e}\left(K^{\varepsilon}\right)$. Let $L$ be a self-adjoint operator and let $\sigma_{p}^{\infty}(L)$ and $\sigma_{c}(L)$ be its set of eigenvalues of infinite multiplicity and its continuous spectrum, respectively. We have $\sigma_{e}(L)=\sigma_{p}^{\infty}(L) \cup \sigma_{c}(L)$ by definition. The spectrum of $K^{\varepsilon}$ is described by the following proposition whose proof is similar to that of [24, Lemma 1].

Lemma 2.14. Let $\rho \in \mathcal{C}_{\text {per }}(Y)$ be such that the sets $\{y \in S: \rho(y)<0\}$ and $\{y \in S: \rho(y)>0\}$ are both of positive surface measure. Then for any $\varepsilon>0$, we have $\sigma\left(K^{\varepsilon}\right) \subset\left[-\left\|K^{\varepsilon}\right\|,\left\|K^{\varepsilon}\right\|\right]$ and $\mu=0$ is the only element of the essential spectrum $\sigma_{e}\left(K^{\varepsilon}\right)$. Moreover, the discrete spectrum of $K^{\varepsilon}$ consists of two infinite sequences

$$
\begin{aligned}
& \mu_{\varepsilon}^{1,+} \geq \mu_{\varepsilon}^{2,+} \geq \cdots \geq \mu_{\varepsilon}^{k,+} \geq \cdots \rightarrow 0^{+}, \\
& \mu_{\varepsilon}^{1,-} \leq \mu_{\varepsilon}^{2,-} \leq \cdots \leq \mu_{\varepsilon}^{k,-} \leq \cdots \rightarrow 0^{-} .
\end{aligned}
$$


Corollary 2.15. The hypotheses are those of Lemma 2.14 Problem (1.2) has a discrete set of eigenvalues consisting of two sequences

$$
\begin{aligned}
& 0<\lambda_{\varepsilon}^{1,+} \leq \lambda_{\varepsilon}^{2,+} \leq \cdots \leq \lambda_{\varepsilon}^{k,+} \leq \cdots \rightarrow+\infty, \\
& 0>\lambda_{\varepsilon}^{1,+} \geq \lambda_{\varepsilon}^{2,-} \geq \cdots \geq \lambda_{\varepsilon}^{k,-} \geq \cdots \rightarrow-\infty .
\end{aligned}
$$

We may now address the homogenization problem for (1.2).

\section{Homogenization results}

In this section we state and prove homogenization results for both cases $M_{S}(\rho)>0$ and $M_{S}(\rho)=0$. The homogenization results in the case when $M_{S}(\rho)<0$ can be deduced from the case $M_{S}(\rho)>0$ by replacing $\rho$ with $-\rho$. We start with the less technical case.

\subsection{The case $M_{S}(\rho)>0$}

We start with the homogenization result for the positive part of the spectrum $\left(\lambda_{\varepsilon}^{k,+}, u_{\varepsilon}^{k,+}\right)_{\varepsilon \in E}$.

\subsubsection{Positive part of the spectrum}

We assume (this is not a restriction) that the corresponding eigenfunctions are orthonormalized as follows

$$
\varepsilon \int_{S^{\varepsilon}} \rho\left(\frac{x}{\varepsilon}\right) u_{\varepsilon}^{k,+} u_{\varepsilon}^{l,+} d \sigma_{\varepsilon}(x)=\delta_{k, l} \quad k, l=1,2, \cdots
$$

and the homogenization results states as

Theorem 3.1. For each $k \geq 1$ and each $\varepsilon \in E$, let $\left(\lambda_{\varepsilon}^{k,+}, u_{\varepsilon}^{k,+}\right)$ be the $k^{\text {th }}$ positive eigencouple to (1.2) with $M_{S}(\rho)>0$ and (3.1). Then, there exists a subsequence $E^{\prime}$ of $E$ such that

$$
\begin{aligned}
& \frac{1}{\varepsilon} \lambda_{\varepsilon}^{k,+} \rightarrow \lambda_{0}^{k} \quad \text { in } \mathbb{R} \text { as } E \ni \varepsilon \rightarrow 0 \\
& P_{\varepsilon} u_{\varepsilon}^{k,+} \rightarrow u_{0}^{k} \quad \text { in } H_{0}^{1}(\Omega) \text {-weak as } E^{\prime} \ni \varepsilon \rightarrow 0 \\
& P_{\varepsilon} u_{\varepsilon}^{k,+} \rightarrow u_{0}^{k} \quad \text { in } L^{2}(\Omega) \text { as } E^{\prime} \ni \varepsilon \rightarrow 0 \\
& \frac{\partial P_{\varepsilon} u_{\varepsilon}^{k,+}}{\partial x_{j}} \stackrel{2 s}{\longrightarrow} \frac{\partial u_{0}^{k}}{\partial x_{j}}+\frac{\partial u_{1}^{k}}{\partial y_{j}} \text { in } L^{2}(\Omega) \text { as } E^{\prime} \ni \varepsilon \rightarrow 0(1 \leq j \leq N)
\end{aligned}
$$

where $\left(\lambda_{0}^{k}, u_{0}^{k}\right) \in \mathbb{R} \times H_{0}^{1}(\Omega)$ is the $k^{\text {th }}$ eigencouple to the spectral problem

$$
\left\{\begin{aligned}
-\sum_{i, j=1}^{N} \frac{\partial}{\partial x_{i}}\left(\frac{1}{M_{S}(\rho)} q_{i j} \frac{\partial u_{0}}{\partial x_{j}}\right) & =\lambda_{0} u_{0} \quad \text { in } \Omega \\
u_{0} & =0 \quad \text { on } \partial \Omega \\
\int_{\Omega}\left|u_{0}\right|^{2} d x & =\frac{1}{M_{S}(\rho)},
\end{aligned}\right.
$$


and where $u_{1}^{k} \in L^{2}\left(\Omega ; H_{p e r}^{1, *}(Y)\right)$. Moreover, for almost every $x \in \Omega$ the following hold true:

(i) The restriction to $Y^{*}$ of $u_{1}^{k}(x)$ is the solution to the variational problem

$$
\left\{\begin{array}{l}
u_{1}^{k}(x) \in H_{p e r}^{1}\left(Y^{*}\right) / \mathbb{R} \\
a\left(u_{1}^{k}(x), v\right)=-\sum_{i, j=1}^{N} \frac{\partial u_{0}^{k}}{\partial x_{j}} \int_{Y^{*}} a_{i j}(y) \frac{\partial v}{\partial y_{i}} d y \\
\forall v \in H_{p e r}^{1}\left(Y^{*}\right) / \mathbb{R} ;
\end{array}\right.
$$

(ii) We have

$$
u_{1}^{k}(x, y)=-\sum_{j=1}^{N} \frac{\partial u_{0}^{k}}{\partial x_{j}}(x) \chi^{j}(y) \quad \text { a.e. in }(x, y) \in \Omega \times Y^{*},
$$

where $\chi^{j}(1 \leq j \leq N)$ is the solution to the cell problem (2.13).

Proof. We present only the outlines since this proof is similar but less technical to that of the case $M_{S}(\rho)=0$.

Fix $k \geq 1$. By means of the minimax principle, as in [35], one easily proves the existence of a constant $C$ independent of $\varepsilon$ such that $\frac{1}{\varepsilon} \lambda_{\varepsilon}^{k,+}<C$. Clearly, for fixed $E \ni \varepsilon>0, u_{\varepsilon}^{k,+}$ lies in $V_{\varepsilon}$, and

$$
\sum_{i, j=1}^{N} \int_{\Omega^{\varepsilon}} a_{i j}\left(\frac{x}{\varepsilon}\right) \frac{\partial u_{\varepsilon}^{k,+}}{\partial x_{j}} \frac{\partial v}{\partial x_{i}} d x=\left(\frac{1}{\varepsilon} \lambda_{\varepsilon}^{k,+}\right) \varepsilon \int_{S^{\varepsilon}} \rho\left(\frac{x}{\varepsilon}\right) u_{\varepsilon}^{k,+} v d \sigma_{\varepsilon}(x)
$$

for any $v \in V_{\varepsilon}$. Bear in mind that $\varepsilon \int_{S^{\varepsilon}} \rho\left(\frac{x}{\varepsilon}\right)\left(u_{\varepsilon}^{k,+}\right)^{2} d x=1$ and choose $v=u_{\varepsilon}^{k,+}$ in (3.9). The boundedness of the sequence $\left(\frac{1}{\varepsilon} \lambda_{\varepsilon}^{k,+}\right)_{\varepsilon \in E}$ and the ellipticity assumption (1.3) imply at once by means of Proposition 2.10 that the sequence $\left(P_{\varepsilon} u_{\varepsilon}^{k,+}\right)_{\varepsilon \in E}$ is bounded in $H_{0}^{1}(\Omega)$. Theorem 2.5 and Proposition 2.8 apply simultaneously and gives us $\mathbf{u}^{k}=\left(u_{0}^{k}, u_{1}^{k}\right) \in \mathbb{F}_{0}^{1}$ such that for some $\lambda_{0}^{k} \in \mathbb{R}$ and some subsequence $E^{\prime} \subset E$ we have (3.2)-(3.5), where (3.4) is a direct consequence of (3.3) by the Rellich-Kondrachov theorem. For fixed $\varepsilon \in E^{\prime}$, let $\Phi_{\varepsilon}$ be as in Lemma 2.12 Multiplying both sides of the first equality in (1.2) by $\Phi_{\varepsilon}$ and integrating over $\Omega^{\varepsilon}$ leads us to the variational $\varepsilon$-problem

$$
\sum_{i, j=1}^{N} \int_{\Omega^{\varepsilon}} a_{i j}\left(\frac{x}{\varepsilon}\right) \frac{\partial P_{\varepsilon} u_{\varepsilon}^{k,+}}{\partial x_{j}} \frac{\partial \Phi_{\varepsilon}}{\partial x_{i}} d x=\left(\frac{1}{\varepsilon} \lambda_{\varepsilon}^{k,+}\right) \varepsilon \int_{S^{\varepsilon}}\left(P_{\varepsilon} u_{\varepsilon}^{k,+}\right) \rho\left(\frac{x}{\varepsilon}\right) \Phi_{\varepsilon} d \sigma_{\varepsilon}(x) .
$$

Sending $\varepsilon \in E^{\prime}$ to 0 , keeping (3.2)-(3.5) and Lemma2.12 in mind, we obtain

$$
\sum_{i, j=1}^{N} \iint_{\Omega \times Y^{*}} a_{i j}(y)\left(\frac{\partial u_{0}^{k}}{\partial x_{j}}+\frac{\partial u_{1}^{k}}{\partial y_{j}}\right)\left(\frac{\partial \psi_{0}}{\partial x_{i}}+\frac{\partial \psi_{1}}{\partial y_{i}}\right) d x d y=\lambda_{0}^{k} \iint_{\Omega \times S} u_{0}^{k} \psi_{0}(x) \rho(y) d x d \sigma(y) .
$$

Therefore, $\left(\lambda_{0}^{k}, \mathbf{u}^{k}\right) \in \mathbb{R} \times \mathbb{F}_{0}^{1}$ solves the following global homogenized spectral problem:

$$
\left\{\begin{array}{l}
\text { Find }(\lambda, \mathbf{u}) \in \mathbb{C} \times \mathbb{F}_{0}^{1} \text { such that } \\
\sum_{i, j=1}^{N} \iint_{\Omega \times Y^{*}} a_{i j}(y)\left(\frac{\partial u_{0}}{\partial x_{j}}+\frac{\partial u_{1}}{\partial y_{j}}\right)\left(\frac{\partial \psi_{0}}{\partial x_{i}}+\frac{\partial \psi_{1}}{\partial y_{i}}\right) d x d y=\lambda M_{S}(\rho) \int_{\Omega} u_{0} \psi_{0} d x \\
\text { for all } \Phi \in \mathbb{F}_{0}^{1} .
\end{array}\right.
$$


which leads to the macroscopic and microscopic problems (3.6)-3.7) without any major difficulty. As regards the normalization condition in (3.6), we fix $k, l \geq 1$ and recall that the following holds for any $\varphi \in \mathcal{D}(\Omega)$ (Proposition 2.8)

$$
\lim _{E^{\prime} \ni \varepsilon \rightarrow 0} \varepsilon \int_{S^{\varepsilon}}\left(P_{\varepsilon} u_{\varepsilon}^{k,+}\right) \varphi(x) \rho\left(\frac{x}{\varepsilon}\right) d \sigma_{\varepsilon}(x)=\iint_{\Omega \times S} u_{0}^{k}(x) \varphi(x) \rho(y) d x d \sigma(y) .
$$

But (3.12) still holds for any $\varphi \in H_{0}^{1}(\Omega)$. This being so, we write

$$
\begin{aligned}
& \varepsilon \int_{S^{\varepsilon}}\left(P_{\varepsilon} u_{\varepsilon}^{k,+}\right)\left(P_{\varepsilon} u_{\varepsilon}^{l,+}\right) \rho\left(\frac{x}{\varepsilon}\right) d \sigma_{\varepsilon}(x)-M_{S}(\rho) \int_{\Omega} u_{0}^{k} u_{0}^{l} d x \\
= & \varepsilon \int_{S^{\varepsilon}}\left(P_{\varepsilon} u_{\varepsilon}^{k,+}\right)\left(P_{\varepsilon} u_{\varepsilon}^{l,+}-u_{0}^{l}\right) \rho\left(\frac{x}{\varepsilon}\right) d \sigma_{\varepsilon}(x)+\varepsilon \int_{S^{\varepsilon}}\left(P_{\varepsilon} u_{\varepsilon}^{k,+}\right) u_{0}^{l} \rho\left(\frac{x}{\varepsilon}\right) d \sigma_{\varepsilon}(x) \\
& -M_{S}(\rho) \int_{\Omega} u_{0}^{k} u_{0}^{l} d x
\end{aligned}
$$

According to (3.12) the sum of the last two terms on the right hand side of (3.13) goes to zero with $\varepsilon \in E^{\prime}$. As the remaining term on the right hand side of (3.13) is concerned, we make use of the Hölder inequality to get

$$
\begin{aligned}
& \left|\varepsilon \int_{S^{\varepsilon}}\left(P_{\varepsilon} u_{\varepsilon}^{k,+}\right)\left(P_{\varepsilon} u_{\varepsilon}^{l,+}-u_{0}^{l}\right) \rho\left(\frac{x}{\varepsilon}\right) d \sigma_{\varepsilon}(x)\right| \\
& \leq\|\rho\|_{\infty}\left(\varepsilon \int_{S^{\varepsilon}}\left|P_{\varepsilon} u_{\varepsilon}^{k,+}\right|^{2} d \sigma_{\varepsilon}(x)\right)^{\frac{1}{2}}\left(\varepsilon \int_{S^{\varepsilon}}\left|P_{\varepsilon} u_{\varepsilon}^{l,+}-u_{0}^{l}\right|^{2} d \sigma_{\varepsilon}(x)\right)^{\frac{1}{2}} .
\end{aligned}
$$

Next the trace inequality (see e.g., [29]) yields

$$
\begin{aligned}
& \varepsilon \int_{S^{\varepsilon}}\left|P_{\varepsilon} u_{\varepsilon}^{k,+}\right|^{2} d \sigma_{\varepsilon}(x) \leq c\left(\int_{\Omega^{\varepsilon}}\left|P_{\varepsilon} u_{\varepsilon}^{k,+}\right|^{2} d x+\varepsilon^{2} \int_{\Omega^{\varepsilon}}\left|D\left(P_{\varepsilon} u_{\varepsilon}^{k,+}\right)\right|^{2} d x\right) \\
& \varepsilon \int_{S^{\varepsilon}}\left|P_{\varepsilon} u_{\varepsilon}^{l,+}-u_{0}^{l}\right|^{2} d \sigma_{\varepsilon}(x) \leq c\left(\int_{\Omega^{\varepsilon}}\left|P_{\varepsilon} u_{\varepsilon}^{l,+}-u_{0}^{l}\right|^{2} d x+\varepsilon^{2} \int_{\Omega^{\varepsilon}}\left|D\left(P_{\varepsilon} u_{\varepsilon}^{l,+}-u_{0}^{l}\right)\right|^{2} d x\right)
\end{aligned}
$$

for some positive constant $c$ independent of $\varepsilon$. But the right hand side of (3.14) is bounded from above whereas that of (3.15) converges to zero with $\varepsilon \in E^{\prime}$. This concludes the proof.

Remark 3.2. - The eigenfunctions $\left\{u_{0}^{k}\right\}_{k=1}^{\infty}$ are in fact orthonormalized as follows

$$
\int_{\Omega} u_{0}^{k} u_{0}^{l} d x=\frac{\delta_{k, l}}{M_{S}(\rho)} \quad k, l=1,2,3, \cdots
$$

- If $\lambda_{0}^{k}$ is simple (this is the case for $\lambda_{0}^{1}$ ), then by Theorem $3.1, \lambda_{\varepsilon}^{k,+}$ is also simple, for small $\varepsilon$, and we can choose the eigenfunctions $u_{\varepsilon}^{k,+}$ such that the convergence results (3.3)-(3.5) hold for the whole sequence $E$.

- Replacing $\rho$ with $-\rho$ in (1.2), Theorem 3.1 also applies to the negative part of the spectrum in the case $M_{S}(\rho)<0$. 


\subsubsection{Negative part of the spectrum}

We now investigate the negative part of the spectrum $\left(\lambda_{\varepsilon}^{k,-}, u_{\varepsilon}^{k,-}\right)_{\varepsilon \in E}$. Before we can do this we need a few preliminaries and stronger regularity hypotheses on $T, \rho$ and the coefficients $\left(a_{i j}\right)_{i, j=1}^{N}$. We assume in this subsection that $\partial T$ is $C^{2, \delta}$ and $\rho$ and the coefficients $\left(a_{i j}\right)_{i, j=1}^{N}$ are $\delta$-Hölder continuous $(0<\delta<1)$.

The following spectral problem is well posed

$$
\left\{\begin{array}{l}
\text { Find }(\lambda, \theta) \in \mathbb{C} \times H_{p e r}^{1}\left(Y^{*}\right) \\
-\sum_{i, j=1}^{N} \frac{\partial}{\partial y_{j}}\left(a_{i j}(y) \frac{\partial \theta}{\partial y_{i}}\right)=0 \text { in } Y^{*} \\
\sum_{i, j=1}^{N} a_{i j}(y) \frac{\partial \theta}{\partial y_{i}} v_{j}=\lambda \rho(y) \theta(y) \text { on } S
\end{array}\right.
$$

and possesses a spectrum with similar properties to that of (1.2), two infinite (one positive and another negative) sequences. We recall that since we have $M_{S}(\rho)>0$, problem 3.16 ) admits a unique nontrivial eigenvalue having an eigenfunction with definite sign, the first negative one (see e.g., [34]). In the sequel we will only make use of $\left(\lambda_{1}^{-}, \theta_{1}^{-}\right)$, the first negative eigencouple to (3.16). After proper sign choice we assume that

$$
\theta_{1}^{-}(y)>0 \text { in } y \in Y^{*} .
$$

We also recall that $\theta_{1}^{-}$is $\delta$-Hölder continuous(see e.g., [14]), hence can be extended to a function living in $\mathcal{C}_{\text {per }}(Y)$ still denoted by $\theta_{1}^{-}$. Notice that we have

$$
\int_{S} \rho(y)\left(\theta_{1}^{-}(y)\right)^{2} d \sigma(y)<0,
$$

as is easily seen from the variational equality (keep the ellipticity hypothesis $(1.3)$ in mind)

$$
\sum_{i, j=1}^{N} \int_{Y^{*}} a_{i j}(y) \frac{\partial \theta_{1}^{-}}{\partial y_{j}} \frac{\partial \theta_{1}^{-}}{\partial y_{i}} d y=\lambda_{1}^{-} \int_{S} \rho(y)\left(\theta_{1}^{-}(y)\right)^{2} d \sigma(y) .
$$

Bear in mind that problem (3.16) induces by a scaling argument the following equalities:

$$
\left\{\begin{array}{l}
-\sum_{i, j=1}^{N} \frac{\partial}{\partial x_{j}}\left(a_{i j}\left(\frac{x}{\varepsilon}\right) \frac{\partial \theta^{\varepsilon}}{\partial x_{i}}\right)=0 \quad \text { in } Q^{\varepsilon} \\
\sum_{i, j=1}^{N} a_{i j}\left(\frac{x}{\varepsilon}\right) \frac{\partial \theta^{\varepsilon}}{\partial x_{i}} v_{j}\left(\frac{x}{\varepsilon}\right)=\frac{1}{\varepsilon} \lambda \rho\left(\frac{x}{\varepsilon}\right) \theta\left(\frac{x}{\varepsilon}\right) \quad \text { on } \partial Q^{\varepsilon},
\end{array}\right.
$$

where $\theta^{\varepsilon}(x)=\theta\left(\frac{x}{\varepsilon}\right)$. However, $\theta^{\varepsilon}$ is not zero on $\partial \Omega$. We now introduce the following Steklov spectral problem (with an indefinite density function)

$$
\left\{\begin{aligned}
\text { Find }\left(\xi_{\varepsilon}, v_{\varepsilon}\right) \in \mathbb{C} \times V_{\varepsilon} & \\
-\sum_{i, j=1}^{N} \frac{\partial}{\partial x_{j}}\left(\widetilde{a}_{i j}\left(\frac{x}{\varepsilon}\right) \frac{\partial v_{\varepsilon}}{\partial x_{i}}\right) & =0 \quad \text { in } \Omega^{\varepsilon} \\
\sum_{i, j=1}^{N} \widetilde{a}_{i j}\left(\frac{x}{\varepsilon}\right) \frac{\partial v_{\varepsilon}}{\partial x_{i}} v_{j}\left(\frac{x}{\varepsilon}\right) & =\xi_{\varepsilon} \widetilde{\rho}\left(\frac{x}{\varepsilon}\right) v_{\varepsilon} \text { on } \partial T^{\varepsilon} \\
v_{\varepsilon}(x) & =0 \text { on } \partial \Omega .
\end{aligned}\right.
$$


with new spectral parameters $\left(\xi_{\varepsilon}, v_{\varepsilon}\right) \in \mathbb{C} \times V_{\varepsilon}$, where $\widetilde{a}_{i j}(y)=\left(\theta_{1}^{-}\right)^{2}(y) a_{i j}(y)$ and $\widetilde{\rho}(y)=$ $\left(\theta_{1}^{-}\right)^{2}(y) \rho(y)$. Notice that $\widetilde{a}_{i j}(y) \in L_{p e r}^{\infty}(Y)$ and $\tilde{\rho}(y) \in \mathcal{C}_{p e r}(Y)$. As $0<c_{-} \leq \theta_{1}^{-}(y) \leq$ $c^{+}<+\infty\left(c_{-}, c^{+} \in \mathbb{R}\right)$, the operator on the left hand side of (3.20) is uniformly elliptic and Theorem 3.1 applies to the negative part of the spectrum of (3.20) (see (3.18) and Remark 3.2). The effective spectral problem for (3.20) reads

$$
\left\{\begin{aligned}
-\sum_{i, j=1}^{N} \frac{\partial}{\partial x_{j}}\left(\widetilde{q}_{i j} \frac{\partial v_{0}}{\partial x_{i}}\right) & =\xi_{0} M_{S}(\widetilde{\rho}) v_{0} \quad \text { in } \Omega \\
v_{0} & =0 \quad \text { on } \partial \Omega \\
\int_{\Omega}\left|v_{0}\right|^{2} d x & =\frac{-1}{M_{S}(\widetilde{\rho})} .
\end{aligned}\right.
$$

The effective coefficients $\left\{\widetilde{q}_{i j}\right\}_{1 \leq i, j \leq N}$ being defined as expected, i.e.,

$$
\widetilde{q}_{i j}=\int_{Y^{*}} \widetilde{a}_{i j}(y) d y-\sum_{l=1}^{N} \int_{Y^{*}} \widetilde{a}_{i l}(y) \frac{\partial \widetilde{\chi}_{1}^{j}}{\partial y_{l}}(y) d y,
$$

with $\widetilde{\chi}^{l} \in H_{\text {per }}^{1}\left(Y^{*}\right) / \mathbb{R}(l=1, \ldots, N)$ being the solution to the following local problem

$$
\left\{\begin{array}{l}
\widetilde{\chi}^{l} \in H_{p e r}^{1}\left(Y^{*}\right) / \mathbb{R} \\
\sum_{i, j=1}^{N} \int_{Y^{*}} \widetilde{a}_{i j}(y) \frac{\partial \widetilde{\chi}^{l}}{\partial y_{j}} \frac{\partial v}{\partial y_{i}} d y=\sum_{i=1}^{N} \int_{Y^{*}} \widetilde{a}_{i l}(y) \frac{\partial v}{\partial y_{i}} d y \\
\text { for all } v \in H_{p e r}^{1}\left(Y^{*}\right) / \mathbb{R} .
\end{array}\right.
$$

We will use the following notation in the sequel:

$$
\widetilde{a}(u, v)=\sum_{i, j=1}^{N} \int_{Y^{*}} \widetilde{a}_{i j}(y) \frac{\partial u}{\partial y_{j}} \frac{\partial v}{\partial y_{i}} d y \quad\left(u, v \in H_{p e r}^{1}\left(Y^{*}\right) / \mathbb{R}\right) .
$$

Notice that the spectrum of (3.21) is as follows

$$
0>\xi_{0}^{1}>\xi_{0}^{2} \geq \xi_{0}^{3} \geq \cdots \geq \xi_{0}^{j} \geq \cdots \rightarrow-\infty \text { as } j \rightarrow \infty .
$$

Making use of (3.19) when following the same line of reasoning as in [35, Lemma 6.1], we obtain that the negative spectral parameters of problems (1.2) and (3.20) verify:

$$
u_{\varepsilon}^{k,-}=\left(\theta_{1}^{-}\right)^{\varepsilon} v_{\varepsilon}^{k,-} \quad(\varepsilon \in E, k=1,2 \cdots)
$$

and

$$
\lambda_{\varepsilon}^{k,-}=\frac{1}{\varepsilon} \lambda_{1}^{-}+\xi_{\varepsilon}^{k,-}+o(1) \quad(\varepsilon \in E, k=1,2 \cdots) .
$$

The presence of the term $o(1)$ is due to integrals over $\Omega^{\varepsilon} \backslash Q^{\varepsilon}$, which converge to zero with $\varepsilon$, remember that $(3.19)$ holds in $Q^{\varepsilon}$ but not $\Omega^{\varepsilon}$. This trick, known as "factorization principle" was introduced by Vaninathan[35] and has been used in many other works on averaging, see e.g., [2, 20, 23] just to cite a few. As will be seen below, the sequence $\left(\xi_{\varepsilon}^{k,-}\right)_{\varepsilon \in E}$ is bounded in $\mathbb{R}$. In another words, $\lambda_{\varepsilon}^{k,-}$ is of order $1 / \varepsilon$ and tends to $-\infty$ as $\varepsilon$ goes to zero. It is 
now clear why the limiting behavior of negative eigencouples is not straightforward as that of positive ones and requires further investigations, which have just been made.

Indeed, as the reader might be guessing now, the suitable orthonormalization condition for 3.20 ) is

$$
\varepsilon \int_{S^{\varepsilon}} \widetilde{\rho}\left(\frac{x}{\varepsilon}\right) v_{\varepsilon}^{k,-} v_{\varepsilon}^{l,-} d \sigma_{\varepsilon}(x)=-\delta_{k, l} \quad k, l=1,2, \cdots
$$

which by means of (3.24) is equivalent to

$$
\varepsilon \int_{S^{\varepsilon}} \rho\left(\frac{x}{\varepsilon}\right) u_{\varepsilon}^{k,-} u_{\varepsilon}^{l,-} d \sigma_{\varepsilon}(x)=-\delta_{k, l} \quad k, l=1,2, \cdots
$$

We may now state the homogenization theorem for the negative part of the spectrum of (1.2).

Theorem 3.3. For each $k \geq 1$ and each $\varepsilon \in E$, let $\left(\lambda_{\varepsilon}^{k,-}, u_{\varepsilon}^{k,-}\right)$ be the $k^{\text {th }}$ negative eigencouple to (1.2) with $M_{S}(\rho)>0$ and (3.27). Then, there exists a subsequence $E^{\prime}$ of $E$ such that

$$
\begin{aligned}
\frac{\lambda_{\varepsilon}^{k,-}}{\varepsilon}-\frac{\lambda_{1}^{-}}{\varepsilon^{2}} & \rightarrow \quad \xi_{0}^{k} \quad \text { in } \mathbb{R} \text { as } E \ni \varepsilon \rightarrow 0 \\
P_{\varepsilon} v_{\varepsilon}^{k,-} & \rightarrow \quad v_{0}^{k} \quad \text { in } H_{0}^{1}(\Omega) \text {-weak as } E^{\prime} \ni \varepsilon \rightarrow 0 \\
P_{\varepsilon} v_{\varepsilon}^{k,-} & \rightarrow \quad v_{0}^{k} \quad \text { in } L^{2}(\Omega) \text { as } E^{\prime} \ni \varepsilon \rightarrow 0 \\
\frac{\partial P_{\varepsilon} v_{\varepsilon}^{k,-}}{\partial x_{j}} & \stackrel{2 s}{\rightarrow} \frac{\partial v_{0}^{k}}{\partial x_{j}}+\frac{\partial v_{1}^{k}}{\partial y_{j}} \text { in } L^{2}(\Omega) \text { as } E^{\prime} \ni \varepsilon \rightarrow 0(1 \leq j \leq N)
\end{aligned}
$$

where $\left(\xi_{0}^{k}, v_{0}^{k}\right) \in \mathbb{R} \times H_{0}^{1}(\Omega)$ is the $k^{\text {th }}$ eigencouple to the spectral problem

$$
\left\{\begin{aligned}
-\sum_{i, j=1}^{N} \frac{\partial}{\partial x_{i}}\left(\frac{1}{M_{S}(\widetilde{\rho})} \widetilde{q}_{i j} \frac{\partial v_{0}}{\partial x_{j}}\right) & =\xi_{0} v_{0} \quad \text { in } \Omega \\
v_{0} & =0 \quad \text { on } \partial \Omega \\
\int_{\Omega}\left|v_{0}\right|^{2} d x & =\frac{-1}{M_{S}(\widetilde{\rho})},
\end{aligned}\right.
$$

and where $v_{1}^{k} \in L^{2}\left(\Omega ; H_{\text {per }}^{1, *}(Y)\right)$. Moreover, for almost every $x \in \Omega$ the following hold true: (i) The restriction to $Y^{*}$ of $v_{1}^{k}(x)$ is the solution to the variational problem

$$
\left\{\begin{array}{l}
v_{1}^{k}(x) \in H_{p e r}^{1}\left(Y^{*}\right) / \mathbb{R} \\
\widetilde{a}\left(v_{1}^{k}(x), u\right)=-\sum_{i, j=1}^{N} \frac{\partial v_{0}^{k}}{\partial x_{j}} \int_{Y^{*}} \widetilde{a}_{i j}(y) \frac{\partial u}{\partial y_{i}} d y \\
\forall u \in H_{p e r}^{1}\left(Y^{*}\right) / \mathbb{R} ;
\end{array}\right.
$$

(ii) We have

$$
v_{1}^{k}(x, y)=-\sum_{j=1}^{N} \frac{\partial v_{0}^{k}}{\partial x_{j}}(x) \tilde{\chi}^{j}(y) \quad \text { a.e. in }(x, y) \in \Omega \times Y^{*},
$$

where $\widetilde{\chi}^{j}(1 \leq j \leq N)$ is the solution to the cell problem 3.23 . 
Remark 3.4. - The eigenfunctions $\left\{v_{0}^{k}\right\}_{k=1}^{\infty}$ are orthonormalized by

$$
\int_{\Omega} v_{0}^{k} v_{0}^{l} d x=\frac{-\delta_{k, l}}{M_{S}(\widetilde{\boldsymbol{\rho}})} \quad k, l=1,2,3, \cdots
$$

- If $\xi_{0}^{k}$ is simple (this is the case for $\xi_{0}^{1}$ ), then by Theorem $3.3, \lambda_{\varepsilon}^{k,-}$ is also simple for small $\varepsilon$, and we can choose the 'eigenfunctions' $v_{\varepsilon}^{k,-}$ such that the convergence results (3.29)-3.31) hold for the whole sequence $E$.

- Replacing $\rho$ with $-\rho$ in (1.2), Theorem 3.3 adapts to the positive part of the spectrum in the case $M_{S}(\rho)<0$.

\subsection{The case $M_{S}(\rho)=0$}

We prove an homogenization result for both the positive part and the negative part of the spectrum simultaneously. We assume in this case that the eigenfunctions are orthonormalized as follows

$$
\int_{S^{\varepsilon}} \rho\left(\frac{x}{\varepsilon}\right) u_{\varepsilon}^{k, \pm} u_{\varepsilon}^{l, \pm} d \sigma_{\varepsilon}(x)= \pm \delta_{k, l} \quad k, l=1,2, \cdots
$$

Let $\chi^{0}$ be the solution to (2.14) and put

$$
v^{2}=\sum_{i, j=1}^{N} \int_{Y^{*}} a_{i j}(y) \frac{\partial \chi^{0}}{\partial y_{j}} \frac{\partial \chi^{0}}{\partial y_{i}} d y .
$$

Indeed, the right hand side of (3.36) is positive. We recall that the following spectral problem for a quadratic operator pencil with respect to $\mathrm{V}$,

$$
\left\{\begin{aligned}
-\sum_{i, j=1}^{N} \frac{\partial}{\partial x_{j}}\left(q_{i j} \frac{\partial u_{0}}{\partial x_{i}}\right) & =\lambda_{0}^{2} v^{2} u_{0} \text { in } \Omega \\
u_{0} & =0 \text { on } \partial \Omega,
\end{aligned}\right.
$$

has a spectrum consisting of two infinite sequences

$$
0<\lambda_{0}^{1,+}<\lambda_{0}^{2,+} \leq \cdots \leq \lambda_{0}^{k,+} \leq \ldots, \quad \lim _{n \rightarrow+\infty} \lambda_{0}^{k,+}=+\infty
$$

and

$$
0>\lambda_{0}^{1,-}>\lambda_{0}^{2,-} \geq \cdots \geq \lambda_{0}^{k,-} \geq \ldots, \quad \lim _{n \rightarrow+\infty} \lambda_{0}^{k,-}=-\infty .
$$

with $\lambda_{0}^{k,+}=-\lambda_{0}^{k,-} \quad k=1,2, \cdots$ and with the corresponding eigenfunctions $u_{0}^{k,+}=u_{0}^{k,-}$. We note by passing that $\lambda_{0}^{1,+}$ and $\lambda_{0}^{1,-}$ are simple. We are now in a position to state the homogenization result in the present case.

Theorem 3.5. For each $k \geq 1$ and each $\varepsilon \in E$, let $\left(\lambda_{\varepsilon}^{k, \pm}, u_{\varepsilon}^{k, \pm}\right)$ be the $(k, \pm)^{\text {th }}$ eigencouple to (1.2) with $M_{S}(\rho)=0$ and (3.35). Then, there exists a subsequence $E^{\prime}$ of $E$ such that

$$
\begin{aligned}
\lambda_{\varepsilon}^{k, \pm} & \rightarrow \lambda_{0}^{k, \pm} \quad \text { in } \mathbb{R} \text { as } E \ni \varepsilon \rightarrow 0 \\
P_{\varepsilon} u_{\varepsilon}^{k, \pm} & \rightarrow u_{0}^{k, \pm} \text { in } H_{0}^{1}(\Omega) \text {-weak as } E^{\prime} \ni \varepsilon \rightarrow 0 \\
P_{\varepsilon} u_{\varepsilon}^{k, \pm} & \rightarrow u_{0}^{k, \pm} \quad \text { in } L^{2}(\Omega) \text { as } E^{\prime} \ni \varepsilon \rightarrow 0 \\
\frac{\partial P_{\varepsilon} u_{\varepsilon}^{k, \pm}}{\partial x_{j}} & \stackrel{2 s}{\rightarrow} \frac{\partial u_{0}^{k, \pm}}{\partial x_{j}}+\frac{\partial u_{1}^{k, \pm}}{\partial y_{j}} \text { in } L^{2}(\Omega) \text { as } E^{\prime} \ni \varepsilon \rightarrow 0(1 \leq j \leq N)
\end{aligned}
$$


where $\left(\lambda_{0}^{k, \pm}, u_{0}^{k, \pm}\right) \in \mathbb{R} \times H_{0}^{1}(\Omega)$ is the $(k, \pm)^{\text {th }}$ eigencouple to the following spectral problem for a quadratic operator pencil with respect to $\mathrm{v}$,

$$
\left\{\begin{aligned}
-\sum_{i, j=1}^{N} \frac{\partial}{\partial x_{i}}\left(q_{i j} \frac{\partial u_{0}}{\partial x_{j}}\right) & =\lambda_{0}^{2} v^{2} u_{0} \text { in } \Omega \\
u_{0} & =0 \text { on } \partial \Omega
\end{aligned}\right.
$$

and where $u_{1}^{k, \pm} \in L^{2}\left(\Omega ; H_{\text {per }}^{1, *}(Y)\right)$. We have the following normalization condition

$$
\int_{\Omega}\left|u_{0}^{k, \pm}\right|^{2} d x=\frac{ \pm 1}{2 \lambda_{0}^{k, \pm} v^{2}} \quad k=1,2, \cdots
$$

Moreover, for almost every $x \in \Omega$ the following hold true:

(i) The restriction to $Y^{*}$ of $u_{1}^{k, \pm}(x)$ is the solution to the variational problem

$$
\left\{\begin{array}{l}
u_{1}^{k, \pm}(x) \in H_{p e r}^{1}\left(Y^{*}\right) / \mathbb{R} \\
a\left(u_{1}^{k, \pm}(x), v\right)=\lambda_{0}^{k, \pm} u_{0}^{k, \pm}(x) \int_{S} \rho(y) v(y) d \sigma(y)-\sum_{i, j=1}^{N} \frac{\partial u_{0}^{k, \pm}}{\partial x_{j}}(x) \int_{Y^{*}} a_{i j}(y) \frac{\partial v}{\partial y_{i}} d y \\
\forall v \in H_{p e r}^{1}\left(Y^{*}\right) / \mathbb{R} ;
\end{array}\right.
$$

(ii) We have

$$
u_{1}^{k, \pm}(x, y)=\lambda_{0}^{k, \pm} u_{0}^{k, \pm}(x) \chi^{0}(y)-\sum_{j=1}^{N} \frac{\partial u_{0}^{k, \pm}}{\partial x_{j}}(x) \chi^{j}(y) \quad \text { a.e. in }(x, y) \in \Omega \times Y^{*},
$$

where $\chi^{j}(1 \leq j \leq N)$ and $\chi^{0}$ are the solutions to the cell problems (2.13) and (2.14), respectively.

Proof. Fix $k \geq 1$, using the minimax principle, as in [35], we get a constant $C$ independent of $\varepsilon$ such that $\left|\lambda_{\varepsilon}^{k, \pm}\right|<C$. We have $u_{\varepsilon}^{k, \pm} \in V_{\varepsilon}$ and

$$
\sum_{i, j=1}^{N} \int_{\Omega^{\varepsilon}} a_{i j}\left(\frac{x}{\varepsilon}\right) \frac{\partial u_{\varepsilon}^{k, \pm}}{\partial x_{j}} \frac{\partial v}{\partial x_{i}} d x=\lambda_{\varepsilon}^{k, \pm} \int_{S^{\varepsilon}} \rho\left(\frac{x}{\varepsilon}\right) u_{\varepsilon}^{k, \pm} v d \sigma_{\varepsilon}(x)
$$

for any $v \in V_{\varepsilon}$. Bear in mind that $\int_{S^{\varepsilon}} \rho\left(\frac{x}{\varepsilon}\right)\left(u_{\varepsilon}^{k, \pm}\right)^{2} d \sigma_{\varepsilon}(x)= \pm 1$ and choose $v=u_{\varepsilon}^{k, \pm}$ in (3.46). The boundedness of the sequence $\left(\lambda_{\varepsilon}^{k, \pm}\right)_{\varepsilon \in E}$ and the ellipticity assumption (1.3) imply at once by means of Proposition 2.10 that the sequence $\left(P_{\varepsilon} u_{\varepsilon}^{k, \pm}\right)_{\varepsilon \in E}$ is bounded in $H_{0}^{1}(\Omega)$. Theorem 2.5 and Proposition 2.8 apply simultaneously and gives us $\mathbf{u}^{k, \pm}=\left(u_{0}^{k, \pm}, u_{1}^{k, \pm}\right) \in \mathbb{F}_{0}^{1}$ such that for some $\lambda_{0}^{k, \pm} \in \mathbb{R}$ and some subsequence $E^{\prime} \subset E$ we have (3.38)-(3.41), where (3.40) is a direct consequence of (3.39) by the Rellich-Kondrachov theorem. For fixed $\varepsilon \in E^{\prime}$, let $\Phi_{\varepsilon}$ be as in Lemma 2.12. Multiplying both sides of the first equality in (1.2) by $\Phi_{\varepsilon}$ and integrating over $\Omega^{\varepsilon}$ leads us to the variational $\varepsilon$-problem

$$
\sum_{i, j=1}^{N} \int_{\Omega^{\varepsilon}} a_{i j}\left(\frac{x}{\varepsilon}\right) \frac{\partial P_{\varepsilon} u_{\varepsilon}^{k, \pm}}{\partial x_{j}} \frac{\partial \Phi_{\varepsilon}}{\partial x_{i}} d x=\lambda_{\varepsilon}^{k, \pm} \int_{S^{\varepsilon}}\left(P_{\varepsilon} u_{\varepsilon}^{k, \pm}\right) \rho\left(\frac{x}{\varepsilon}\right) \Phi_{\varepsilon} d \sigma_{\varepsilon}(x) .
$$


Sending $\varepsilon \in E^{\prime}$ to 0 , keeping (3.38)-(3.41) and Lemma2.12 in mind, we obtain

$$
a_{\Omega}\left(\mathbf{u}^{k, \pm}, \Phi\right)=\lambda_{0}^{k, \pm} \iint_{\Omega \times S}\left(u_{1}^{k, \pm}(x, y) \psi_{0}(x) \rho(y)+u_{0}^{k, \pm} \psi_{1}(x, y) \rho(y)\right) d x d \sigma(y)
$$

The right-hand side follows as explained below. we have

$$
\begin{aligned}
\int_{S^{\varepsilon}}\left(P_{\varepsilon} u_{\varepsilon}^{k, \pm}\right) \rho\left(\frac{x}{\varepsilon}\right) \Phi_{\varepsilon} d \sigma_{\varepsilon}(x) & =\int_{S^{\varepsilon}}\left(P_{\varepsilon} u_{\varepsilon}^{k, \pm}\right) \psi_{0}(x) \rho\left(\frac{x}{\varepsilon}\right) d \sigma_{\varepsilon}(x) \\
& +\varepsilon \int_{S^{\varepsilon}}\left(P_{\varepsilon} u_{\varepsilon}^{k, \pm}\right) \psi_{1}\left(x, \frac{x}{\varepsilon}\right) \rho\left(\frac{x}{\varepsilon}\right) d \sigma_{\varepsilon}(x)
\end{aligned}
$$

On the one hand we have

$$
\lim _{E^{\prime} \ni \varepsilon \rightarrow 0} \varepsilon \int_{S^{\varepsilon}}\left(P_{\varepsilon} u_{\varepsilon}^{k, \pm}\right) \psi_{1}\left(x, \frac{x}{\varepsilon}\right) \rho\left(\frac{x}{\varepsilon}\right) d x=\iint_{\Omega \times S} u_{0}^{k, \pm} \psi_{1}(x, y) \rho(y) d x d \sigma(y) .
$$

On the other hand, owing to Lemma 2.9, the following holds:

$$
\lim _{E^{\prime} \ni \varepsilon \rightarrow 0} \int_{S^{\varepsilon}}\left(P_{\varepsilon} u_{\varepsilon}^{k, \pm}\right) \psi_{0}(x) \rho\left(\frac{x}{\varepsilon}\right) d \sigma_{\varepsilon}(x)=\iint_{\Omega \times S} u_{1}^{k, \pm}(x, y) \psi_{0}(x) \rho(y) d x d \sigma(y) .
$$

We have just proved that $\left(\lambda_{0}^{k, \pm}, \mathbf{u}^{k, \pm}\right) \in \mathbb{R} \times \mathbb{F}_{0}^{1}$ solves the following global homogenized spectral problem:

$$
\left\{\begin{array}{l}
\text { Find }(\lambda, \mathbf{u}) \in \mathbb{C} \times \mathbb{F}_{0}^{1} \text { such that } \\
a_{\Omega}(\mathbf{u}, \Phi)=\lambda \iint_{\Omega \times S}\left(u_{1}(x, y) \psi_{0}(x)+u_{0}(x) \psi_{1}(x, y)\right) \rho(y) d x d \sigma(y) \\
\text { for all } \Phi \in \mathbb{F}_{0}^{1} .
\end{array}\right.
$$

To prove (i), choose $\Phi=\left(\psi_{0}, \psi_{1}\right)$ in 3.47) such that $\psi_{0}=0$ and $\psi_{1}=\varphi \otimes v_{1}$, where $\varphi \in \mathcal{D}(\Omega)$ and $v_{1} \in H_{p e r}^{1}\left(Y^{*}\right) / \mathbb{R}$ to get

$$
\int_{\Omega} \varphi(x)\left[\sum_{i, j=1}^{N} \int_{Y^{*}} a_{i j}(y)\left(\frac{\partial u_{0}^{k, \pm}}{\partial x_{j}}+\frac{\partial u_{1}^{k, \pm}}{\partial y_{j}}\right) \frac{\partial v_{1}}{\partial y_{i}} d y\right] d x=\int_{\Omega} \varphi(x)\left[\lambda_{0}^{k, \pm} u_{0}^{k, \pm}(x) \int_{S} v_{1}(y) \rho(y) d \sigma(y)\right] d x
$$

Hence by the arbitrariness of $\varphi$, we have a.e. in $\Omega$

$$
\sum_{i, j=1}^{N} \int_{Y^{*}} a_{i j}(y)\left(\frac{\partial u_{0}^{k, \pm}}{\partial x_{j}}+\frac{\partial u_{1}^{k, \pm}}{\partial y_{j}}\right) \frac{\partial v_{1}}{\partial y_{i}} d y=\lambda_{0}^{k, \pm} u_{0}^{k, \pm}(x) \int_{S} v_{1}(y) \rho(y) d \sigma(y)
$$

for any $v_{1}$ in $H_{p e r}^{1}\left(Y^{*}\right) / \mathbb{R}$, which is nothing but (3.44).

Fix $x \in \bar{\Omega}$, multiply both sides of 2.13 by $-\frac{\partial u_{0}^{k, \pm}}{\partial x_{j}}(x)$ and sum over $1 \leq j \leq N$. Adding side by side to the resulting equality that obtained after multiplying both sides of (2.14) by $\lambda_{0}^{k, \pm} u_{0}^{k, \pm}(x)$, we realize that $z(x)=-\sum_{j=1}^{N} \frac{\partial u_{0}^{k, \pm}}{\partial x_{j}}(x) \chi^{j}(y)+\lambda_{0}^{k, \pm} u_{0}^{k, \pm}(x) \chi^{0}(y)$ solves (3.44). Hence

$$
u_{1}^{k, \pm}(x, y)=\lambda_{0}^{k, \pm} u_{0}^{k, \pm}(x) \chi^{0}(y)-\sum_{j=1}^{N} \frac{\partial u_{0}^{k, \pm}}{\partial x_{j}}(x) \chi^{j}(y) \text { a.e. in } \Omega \times Y^{*},
$$


by uniqueness of the solution to (3.44). Thus (3.45). But (3.49) still holds almost everywhere in $(x, y) \in \Omega \times S$ as $S$ is of class $C^{1}$. Considering now $\Phi=\left(\psi_{0}, \psi_{1}\right)$ in (3.47) such that $\psi_{0} \in \mathcal{D}(\Omega)$ and $\psi_{1}=0$ we get

$$
\sum_{i, j=1}^{N} \iint_{\Omega \times Y^{*}} a_{i j}(y)\left(\frac{\partial u_{0}^{k, \pm}}{\partial x_{j}}+\frac{\partial u_{1}^{k, \pm}}{\partial y_{j}}\right) \frac{\partial \psi_{0}}{\partial x_{i}} d x d y=\lambda_{0}^{k, \pm} \iint_{\Omega \times S} u_{1}^{k, \pm}(x, y) \rho(y) \psi_{0}(x) d x d \sigma(y),
$$

which by means of 3.49 leads to

$$
\begin{aligned}
& \sum_{i, j=1}^{N} \int_{\Omega} q_{i j} \frac{\partial u_{0}^{k, \pm}}{\partial x_{j}} \frac{\partial \psi_{0}}{\partial x_{i}} d x+\lambda_{0}^{k, \pm} \sum_{i, j=1}^{N} \int_{\Omega} u_{0}^{k, \pm}(x) \frac{\partial \psi_{0}}{\partial x_{i}}\left(\int_{Y^{*}} a_{i j}(y) \frac{\partial \chi^{0}}{\partial y_{j}}(y) d y\right) d x \\
& =-\lambda_{0}^{k, \pm} \sum_{j=1}^{N} \int_{\Omega} \frac{\partial u_{0}^{k, \pm}}{\partial x_{j}} \psi_{0}(x)\left(\int_{S} \rho(y) \chi^{j}(y) d \sigma(y)\right) d x \\
& +\left(\lambda_{0}^{k, \pm}\right)^{2} \int_{\Omega} u_{0}^{k, \pm}(x) \psi_{0}(x)\left(\int_{S} \rho(y) \chi^{0}(y) d \sigma(y)\right) d x .
\end{aligned}
$$

Choosing $\chi^{l}(1 \leq l \leq N)$ as test function in (2.14) and $\chi^{0}$ as test function in (2.13) we observe that

$$
\sum_{j=1}^{N} \int_{Y^{*}} a_{l j}(y) \frac{\partial \chi^{0}}{\partial y_{j}}(y) d y=\int_{S} \rho(y) \chi^{l}(y) d \sigma(y)=a\left(\chi^{l}, \chi^{0}\right) \quad(l=1, \cdots N) .
$$

Thus, in 3.50, the second term in the left hand side is equal to the first one in the right hand side. This leaves us with

$$
\int_{\Omega} q_{i j} \frac{\partial u_{0}^{k, \pm}}{\partial x_{j}} \frac{\partial \psi_{0}}{\partial x_{i}} d x=\left(\lambda_{0}^{k, \pm}\right)^{2} \int_{\Omega} u_{0}^{k, \pm}(x) \psi_{0}(x) d x\left(\int_{S} \rho(y) \chi^{0}(y) d \sigma(y)\right) .
$$

Choosing $\chi^{0}$ as test function in (2.14) reveals that

$$
\int_{S} \rho(y) \chi^{0}(y) d \sigma(y)=a\left(\chi^{0}, \chi^{0}\right)=v^{2} .
$$

Hence

$$
\sum_{i, j=1}^{N} \int_{\Omega} q_{i j} \frac{\partial u_{0}^{k, \pm}}{\partial x_{j}} \frac{\partial \psi_{0}}{\partial x_{i}} d x=\left(\lambda_{0}^{k, \pm}\right)^{2} v^{2} \int_{\Omega} u_{0}^{k, \pm}(x) \psi_{0}(x) d x
$$

and

$$
-\sum_{i, j=1}^{N} \frac{\partial}{\partial x_{i}}\left(q_{i j} \frac{\partial u_{0}^{k, \pm}}{\partial x_{j}}(x)\right)=\left(\lambda_{0}^{k, \pm}\right)^{2} v^{2} u_{0}^{k, \pm}(x) \text { in } \Omega .
$$

Thus, the convergence (3.38) holds for the whole sequence $E$. We now address 3.43). Fix $k, l \geq 1$ and let $\vartheta \in H_{p e r}^{1}\left(Y^{*}\right) / \mathbb{R}$ be the solution to (2.10) where $\theta$ is replaced with our density function $\rho$. As in (2.11), we transform the surface integral into a volume integral

$$
\begin{aligned}
\int_{S^{\varepsilon}}\left(P_{\varepsilon} u_{\varepsilon}^{k, \pm}\right)\left(P_{\varepsilon} u_{\varepsilon}^{l, \pm}\right) \rho\left(\frac{x}{\varepsilon}\right) d \sigma_{\varepsilon}(x) & =\int_{\Omega^{\varepsilon}}\left(P_{\varepsilon} u_{\varepsilon}^{k, \pm}\right) D_{x}\left(P_{\varepsilon} u_{\varepsilon}^{l, \pm}\right) \cdot D_{y} \vartheta\left(\frac{x}{\varepsilon}\right) d x \\
& +\int_{\Omega^{\varepsilon}} D_{x}\left(P_{\varepsilon} u_{\varepsilon}^{k, \pm}\right)\left(P_{\varepsilon} u_{\varepsilon}^{l, \pm}\right) \cdot D_{y} \vartheta\left(\frac{x}{\varepsilon}\right) d x .
\end{aligned}
$$


A limit passage in (3.52) as $E^{\prime} \ni \varepsilon \rightarrow 0$ yields

$$
\begin{aligned}
& \lim _{E^{\prime \ni \varepsilon \rightarrow 0}} \int_{S^{\varepsilon}}\left(P_{\varepsilon} u_{\varepsilon}^{k, \pm}\right)\left(P_{\varepsilon} u_{\varepsilon}^{l, \pm}\right) \rho\left(\frac{x}{\varepsilon}\right) d \sigma_{\varepsilon}(x) \\
= & \iint_{\Omega \times Y^{*}} u_{0}^{k, \pm}\left(D_{x} u_{0}^{l, \pm}+D_{y} u_{1}^{l, \pm}\right) \cdot D_{y} \vartheta d x d y+\iint_{\Omega \times Y^{*}}\left(D_{x} u_{0}^{k, \pm}+D_{y} u_{1}^{k, \pm}\right) u_{0}^{l, \pm} \cdot D_{y} \vartheta d x d y \\
= & \int_{\Omega} u_{0}^{k, \pm}\left(\int_{Y^{*}} D_{y} u_{1}^{l, \pm}(x, y) \cdot D_{y} \vartheta(y) d y\right) d x+\int_{\Omega} u_{0}^{l, \pm}\left(\int_{Y^{*}} D_{y} u_{1}^{k, \pm}(x, y) \cdot D_{y} \vartheta(y) d y\right) d x \\
= & \iint_{\Omega \times S} u_{0}^{k, \pm}(x) u_{1}^{l, \pm}(x, y) \rho(y) d x d \sigma(y)+\iint_{\Omega \times S} u_{0}^{l, \pm}(x) u_{1}^{k, \pm}(x, y) \rho(y) d x d \sigma(y) \\
= & \lambda_{0}^{l, \pm} v^{2} \int_{\Omega} u_{0}^{k, \pm}(x) u_{0}^{l, \pm}(x) d x+\lambda_{0}^{k, \pm} v^{2} \int_{\Omega} u_{0}^{l, \pm}(x) u_{0}^{k, \pm}(x) d x \\
= & \left(\lambda_{0}^{k, \pm}+\lambda_{0}^{l, \pm}\right) v^{2} \int_{\Omega} u_{0}^{k, \pm}(x) u_{0}^{l, \pm}(x) d x .
\end{aligned}
$$

Where after the limit passage, we used the integration by part formula, then the weak formulation of (2.10) and finally (3.45) and integration by part. If $k=l$, the above limit passage and (3.35) lead to the desired result, (3.43), completing thereby the proof.

Remark 3.6. - The eigenfunctions $\left\{u_{0}^{k, \pm}\right\}_{k=1}^{\infty}$ are in fact orthonormalized as follows

$$
\int_{\Omega} u_{0}^{l, \pm}(x) u_{0}^{k, \pm}(x) d x=\frac{ \pm \delta_{k, l}}{v^{2}\left(\lambda_{0}^{l, \pm}+\lambda_{0}^{k, \pm}\right)} \quad k, l=1,2, \cdots
$$

- If $\lambda_{0}^{k, \pm}$ is simple (this is the case for $\lambda_{0}^{1, \pm}$ ), then by Theorem $3.5, \lambda_{\varepsilon}^{k, \pm}$ is also simple, for small $\varepsilon$, and we can choose the eigenfunctions $u_{\varepsilon}^{k, \pm}$ such that the convergence results (3.39)-3.41) hold for the whole sequence $E$.

\section{Final Remark}

After this paper was completed (see [9]) and submitted, we learned about an independent and similar work [6].

\section{Acknowledgments}

The author is grateful to Dr. Jean Louis Woukeng for helpful discussions.

\section{References}

[1] G. Allaire, Homogenization and two-scale convergence, SIAM J. Math. Anal., 23 (1992), 1482-1518.

[2] G. Allaire and A. Piatnitski, Uniform spectral asymptotics for singularly perturbed locally periodic operators, Comm. Partial Differential Equations, 27 (2002), 705-725. 
[3] G. Allaire, A. Damlamian and U. Hornung, Two-scale convergence on periodic surfaces and applications, In Proceedings of the International Conference on Mathematical Modelling of Flow through Porous Media (May 1995), A. Bourgeat et al. eds., pp.15-25, World Scientific Pub., Singapore (1996).

[4] A. Bensoussan, J.L. Lions and G. Papanicolaou, "Asymptotic analysis for periodic structures", North-Holland, Amsterdam, 1978.

[5] M.S. Birman, M.Z. Solomyack, "Spectral theory of self-adjoint operators in Hilbert spaces”, D. Reidel Publishing Company, Dordrecht, 1987.

[6] V. Chiado piat, S.S. Nazarov and A.L. Piatnitski, Steklov problems in perforated domains with a coefficient of indefinite sign, Network and Heteregeneous media, 7 (2012), 151-178.

[7] D. Cioranescu and P. Donato, Homogénéisation du problème de Neuman non homogène dans des ouverts perforé, Asymptotic Anal., 1 (1988), 115-138.

[8] D. Cioranescu and J. Saint Jean Paulin, Homogenization in open sets with holes, J. Math. Appl., 71 (1979), 590-607.

[9] H. Douanla, Homogenization of Steklov spectral problems with indefinite indefinite density function in perforated domains, Preprint, June 2011, arXiv:1106.3904v1.

[10] H. Douanla, Two-Scale convergence of elliptic spectral problems with indefinite density function in perforated domains, Preprint, June 2011, arXiv:1106.3907v1. To appear in Asymptotic Analysis.

[11] H. Douanla, Two-Scale convergence of Stekloff eigenvalue problems in perforated domains, Boundary Value Problems, 2010 (2010), Article ID 853717, 15 pages.

[12] H. Douanla and N. Svanstedt, Reiterated homogenization of linear eigenvalue problems in multiscale perforated domains beyond the periodic setting, Commun. Math. Anal. 11 (2011), no. 1, 61-93.

[13] D. G. Figueiredo, Positive solutions of semilinear elliptic problems, Lecture Notes in Maths.,954, Springer-Verlag, Berlin (1982), 34-87.

[14] D. Gilbarg and N.S Trudinger, Elliptic partial differential equations of second order, Classics in Mathematics, Springer, Berlin (2001), 34-87.

[15] P. Hess, On bifurcation from infinity for positive solutions of second order elliptic eigenvalue problems, Nonlinear Phenomena in Mathematical Sciences., Academic Press, New York, (1982), 537-5

[16] P. Hess and T. Kato, On some linear and nonlinear eigenvalue problems with an indefinite weight function, Comm. Partial. Differential Equations., 5 (1980), 9991030 . 
[17] S. Kaizu, Homogenization of eigenvalue problems for the laplace operators with nonlinear terms in domains in many tiny holes, Nonlin. Anal. TMA., 28 (1997), 377391.

[18] S. Kesavan, Homogenization of elliptic eigenvalue problems. I., Appl. Math. Optim., 5 (1979), 153-167.

[19] S. Kesavan, Homogenization of elliptic eigenvalue problems. II., Appl. Math. Optim., 5 (1979), 197-216.

[20] S. M. Kozlov, Reducibility of quasi periodic differential operators and averaging, (Russian) Trudy Moskov. Mat. Obshch., 46 (1983), 99-123.

[21] D. Lukkassen, G. Nguetseng and P. Wall, Two-scale convergence, Int. J. Pure Appl. Math., 2 (2002), 35-86.

[22] S.A. Nazarov, Asymptotics of negative eigenvalues of the Dirichlet problem with the density changing sign, J. Math. Sci., 163 (2009), 151-175.

[23] S.A. Nazarov, A.L. Piatnitski, Homogenization of the spectral Dirichlet problem for a system of differential equations with rapidly oscillating coefficients and changing sing density, J. Math. Sci., 169 (2010), 212-248.

[24] S.A. Nazarov, I.L. Pankratova, A.L. Piatnitski, Homogenization of the spectral problem for periodic elliptic operators with sign-changing density function, Arch. Rational Mech. Anal., DOI: 10.1007/s00205-010-0370-2.

[25] G. Nguetseng, A general convergence result for a functional related to the theory of homogenization, SIAM J. Math. Anal., 20, (1989), 608-623.

[26] G. Nguetseng, Homogenization in perforated domains beyond the periodic setting, J. Math. Anal. Appl., 289 (2004), 608-628.

[27] G. Nguetseng, J.L. Woukeng, $\Sigma$-convergence of nonlinear parabolic operators, Nonlinear Anal., 66 (2007), 968-1004.

[28] O. A. Oleinik, G. A. Yosifian and A. S. Shamaev, Mathematical problems of the theory of strongly nonhomogeneous elastic media [in Russian], Moscow, 1990.

[29] S. E. Pastukhova, Averaging error for the Steklov problem in a perforated domain, Differential Equations, 31 (1995), 975-986.

[30] M. Radu, Homogenization techniques, Diplomarbeit, University of Heidelberg: Faculty of Mathematics, July 1992.

[31] M. Radu, Some extensions of two-scale convergence, C. R. Acad. Sci. Paris Sér. I Math., 9 (1996), 899-904.

[32] S. Roppongi, Asymptotics of eigenvalues of the Laplacian with small spherical Robin boundary, Osaka J. Math., 30 (1993), 783-811. 
[33] M. V. Steklov, Sur les problèmes fondamentaux de la physique mathématique, Ann. Sci. Ecole normale Sup., 19 (1902), 455-490.

[34] O. Torne, Steklov problem with an indefinite weight for the p-laplacian, Elecron. J. Differ. Equ., 87 (2005), 1-8.

[35] M. Vanninathan, Homogenization of eigenvalue problems in perforated domains, Proc. Indian Acad. Sci. (Math. Sci.), 90 (1981), 239-271.

[36] V.V. Zhikov, On two-scale convergence. (Russian) Tr. Semin. im. I. G. Petrovskogo No. 23 (2003), 149-187, 410; translation in J. Math. Sci. (N. Y.) 120 (2004), no. 3, $1328-1352$ 